\title{
Structure-Guided Drug Design of 6-Substituted Adenosine Analogs as Potent Inhibitors of Mycobacterium tuberculosis Adenosine Kinase
}

Roberto A. Crespo ${ }^{\dagger \infty}$, Qun Dang ${ }^{\ddagger} \|$, Nian E. Zhou ${ }^{\dagger}$, Liam M. Guthrie ${ }^{+}$, Thomas C. Snavely ${ }^{\dagger}$, Wen Dong $^{\dagger}$, Kimberly A. Loesch ${ }^{\dagger}$, Takao Suzuki\#, Lanying You\#, Wei Wang\#, Theresa O’Malley ${ }^{\circ}$, Tanya Parish $^{\circ}$, David B. Olsen ${ }^{\ddagger *}$ and James C. Sacchettini ${ }^{\dagger *}$

†Department of Biochemistry and Biophysics, Texas A\&M University, College Station, TX, 77843, US.

${ }^{+}$College of Medicine, Texas A\&M University Health Science Center, Bryan, TX, 77807, US.

${ }^{\ddagger}$ Merck Sharp Dohme Corp., West Point PA, 19486.

\#WuXi AppTec, 288 Fute Zhong Road, Shanghai 200131 China.

${ }^{\circ}$ TB Discovery Research, Infectious Disease Research Institute, 1616 Eastlake Avenue E, Seattle, WA 98102, USA.

Supporting Information 


\section{TABLE OF CONTENTS}

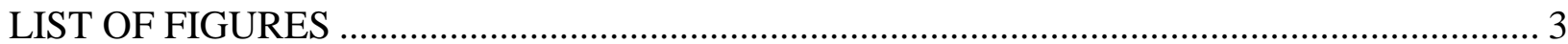

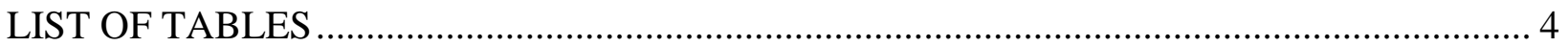

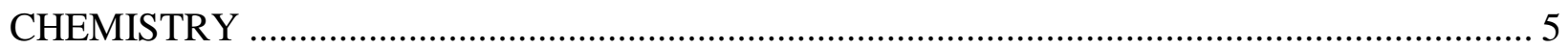

Schemes and reagaents for chemical synthesis of intermediates ......................................... 5

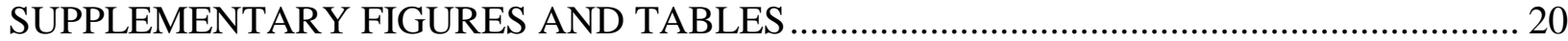

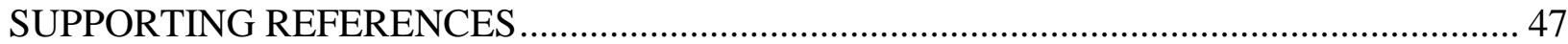




\section{LIST OF FIGURES}

Figure S1. Superimposition of the closed and open conformations of MtbAdoK.................... 20 Figure S2. MtbAdoK homodimer embedded with two copies of compound 2 and compound 3 per

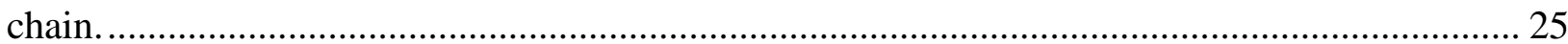

Figure S3. MtbAdoK dimer bound to two copies of compound 4 per chain.......................... 29

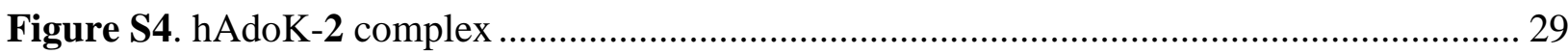

Figure S5. MtbAdoK-2 structure showing the relative position of the iodotubercidin bound to the

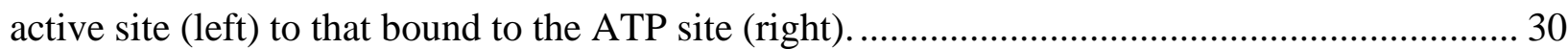

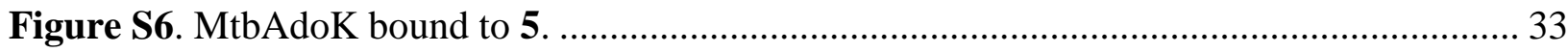

Figure S7. MtbAdoK dimer with two molecules of compound $\mathbf{6}$ per chain.............................. 36

Figure S8. Dose-response curve of compound $\mathbf{6}$ when tested against $\mathrm{mc}^{2} 7000 \ldots \ldots \ldots \ldots \ldots \ldots \ldots . . . . . . . . .37$

Figure S9. Dose-response curve of compound $\mathbf{6}$ when tested against HDF cells...................... 37

Figure S10. MtbAdoK dimer with two molecules of compound 7 per chain........................... 40

Figure S11. Chemical structures of synthesized adenosine analogs with substitutions at the 5'-

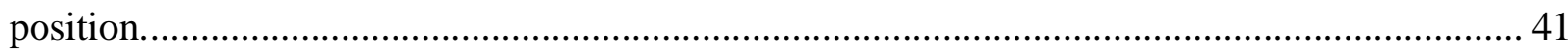

Figure S12. MtbAdoK dimer with two molecules of compound 17 per chain.......................... 44

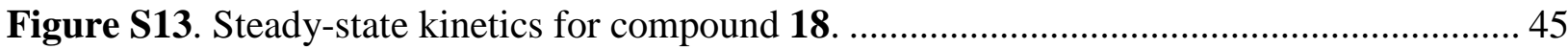




\section{LIST OF TABLES}

Table S1. Close contacts of the MtbAdoK-2 complex $\leq 3.5 \AA$. .......................................... 21

Table S2. Crystal data collection and refinement statistics for MtbAdoK-2 ............................ 22

Table S3. Close contacts of the MtbAdoK-3 complex $\leq 3.5 \AA$............................................. 23

Table S4. Crystal data collection and refinement statistics for MtbAdoK-3. ........................... 24

Table S5. Close contacts of the MtbAdoK-1 complex $\leq 3.5 \AA$............................................. 26

Table S6. Close contacts of the MtbAdoK-4 complex $\leq 3.5 \AA$............................................ 27

Table S7. Crystal data collection and refinement statistics for MtbAdoK-4 ........................... 28

Table S8. Close contacts of the MtbAdoK-5 complex $\leq 3.5 \AA$.......................................... 31

Table S9. Crystal data collection and refinement statistics for MtbAdoK-5 .......................... 32

Table S10. Close contacts of the MtbAdoK-6 complex $\leq 3.5 \AA \AA$............................................ 34

Table S11. Crystal data collection and refinement statistics for MtbAdoK-6......................... 35

Table S12. Close contacts of the MtbAdoK-7 complex $\leq 3.5 \AA \AA$........................................... 38

Table S13. Crystal data collection and refinement statistics for MtbAdoK-7.......................... 39

Table S14. SAR data for synthesized adenosine analogs with substitutions at the 5'-position... 41

Table S15. Close contacts of the MtbAdoK-17 complex $\leq 3.5 \AA$. .......................................... 42

Table S16. Crystal data collection and refinement statistics for MtbAdoK-17...................... 43

Table S17. Steady-state kinetic parameters for MtbAdoK vs. compound 18........................... 45

Table S18. Mouse Acute Tolerability Studies. .................................................................... 46 


\section{CHEMISTRY}

Schemes and reagaents for chemical synthesis of intermediates

Scheme A0: Synthesis of (2R,3R,4S,5R)-2-(7-(4-([1-phenyl]-4-yl)piperazin-1-yl)-3Himidazo[4,5-b]pyridin-3-yl)-5-(hydroxymethyl)tetrahydrofuran-3,4-diol (7)

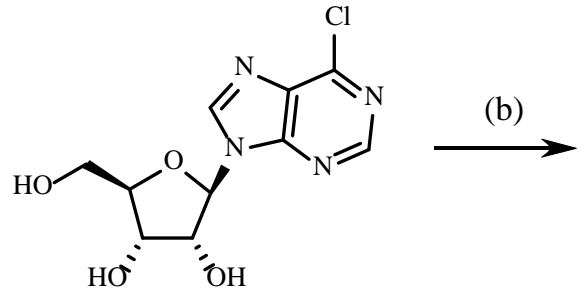

A0

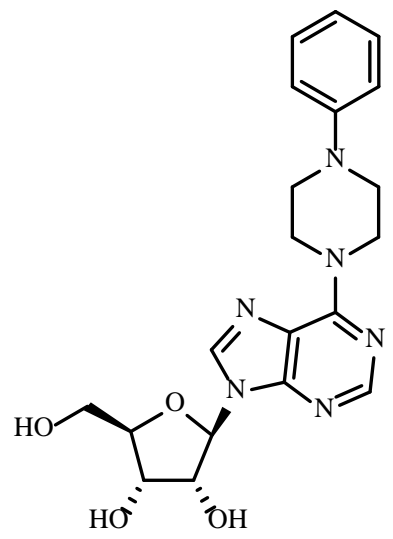

7

Reagents: (b) 1-([1-phenyl]-4-yl)piperazine, DIEA, EtOH, $70{ }^{\circ} \mathrm{C}, 17 \mathrm{~h}$. 
Scheme A: Synthesis of (2R,3R,4S,5R)-2-(6-(4-([1,1'-biphenyl]-4-yl)piperazin-1-yl)-9Hpurin-9-yl)-5-(hydroxymethyl)tetrahydrofuran-3,4-diol (18)

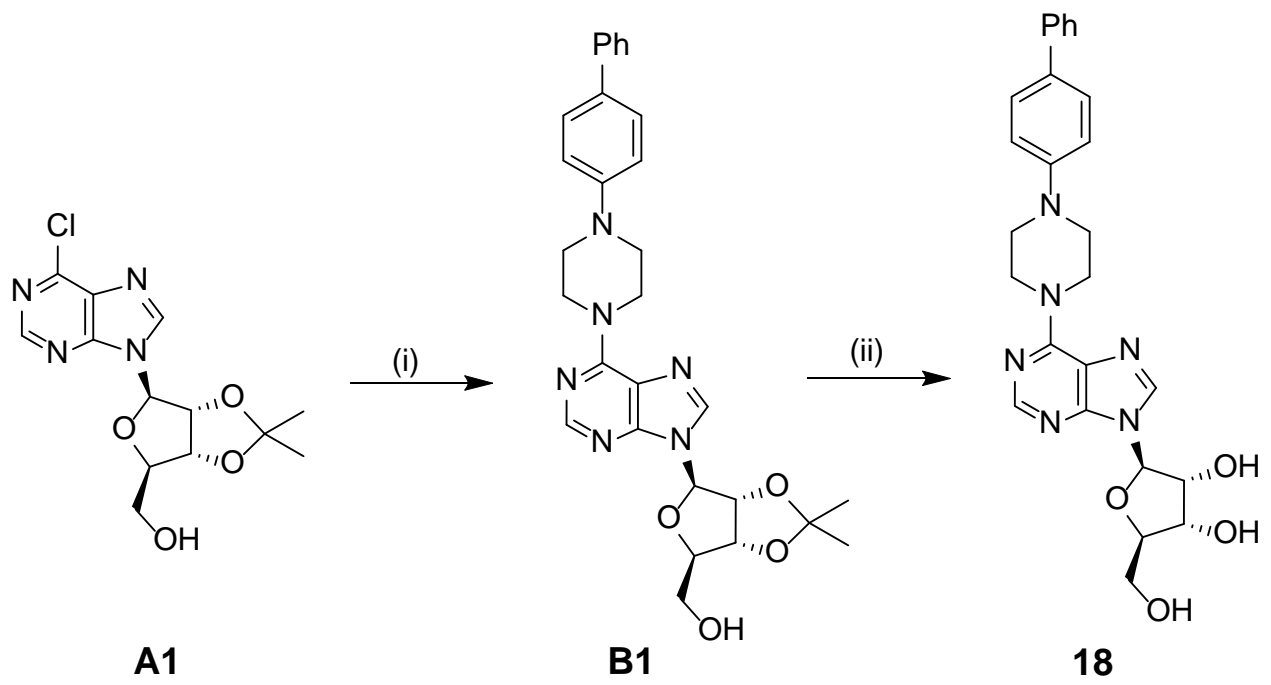

Reagents: (i) 1-([1,1'-biphenyl]-4-yl)piperazine hydrochloride, DIEA, EtOH, $80^{\circ} \mathrm{C}, 17 \mathrm{~h}$; (ii) TFA, THF/ $\mathrm{H}_{2} \mathrm{O}, 15^{\circ} \mathrm{C}, 2 \mathrm{~h}$.

((3aR,4R,6R,6aR)-6-(6-(4-([1,1'-biphenyl]-4-yl)piperazin-1-yl)-9H-purin-9-yl)-2,2dimethyltetrahydrofuro[3,4-d][1,3]dioxol-4-yl)methanol (B1)

To a suspension of 1-([1,1'-biphenyl]-4-yl) piperazine hydrochloride (2.7 g, $8.84 \mathrm{mmol})$ in anhydrous EtOH (70 mL) were added DIEA (4.28 mL, $24.48 \mathrm{mmol})$ and ((3aR,4R,6R,6aR)-6-(6chloro-9H-purin-9-yl)-2,2-dimethyltetrahydrofuro[3,4-d][1,3]dioxol-4-yl)methanol A1 (2.0 g, $6.12 \mathrm{mmol})^{1}$ at room temperature. After it was stirred at $80^{\circ} \mathrm{C}$ for $17 \mathrm{~h}$, the mixture was diluted with EtOH $(30 \mathrm{~mL})$, filtered. The resulting precipitate was washed with EtOH $(20 \mathrm{~mL} \times 3)$, dried under reduced pressure to afford ((3aR,4R,6R,6aR)-6-(6-(4-([1,1'-biphenyl]-4-yl)piperazin-1-yl)9H-purin-9-yl)-2,2-dimethyltetrahydrofuro[3,4-d][1,3]dioxol-4-yl)methanol B1 (3.1 g, 90\% yield) as a white solid. ${ }^{1} \mathrm{H}$ NMR (DMSO- $\left.d_{6}, 400 \mathrm{MHz}\right) \delta 8.44$ (s, $1 \mathrm{H}$ ), 8.31 (s, $\left.1 \mathrm{H}\right), 7.61-7.55$ (m, 4H), 7.43-7.39 (m, 2H), 7.29-7.27 (m, 1H), 7.09 (d, $J=8.4$ Hz, 2H), 6.17 (d, $J=2.0 \mathrm{~Hz}, 1 \mathrm{H}$ ), 5.35-5.34 (m, 1H), 5.23 (t, $J=4.8 \mathrm{~Hz}, 1 \mathrm{H}$ ), 4.98-4.97 (m, 1H), 4.39 (brs, 4H), 4.24 (s, 1H), 3.59-3.52 (m, 2H), 3.35 (s, 4H), 1.51 (s, 3H), 1.28 (s, 3H). MS (ESI) m/z: 529.4 [M+H] . 
Scheme B: Synthesis of (2R,3R,4S,5R)-2-(7-(4-([1,1'-biphenyl]-4-yl)piperazin-1-yl)-3Himidazo[4,5-b]pyridin-3-yl)-5-(hydroxymethyl)tetrahydrofuran-3,4-diol (8)

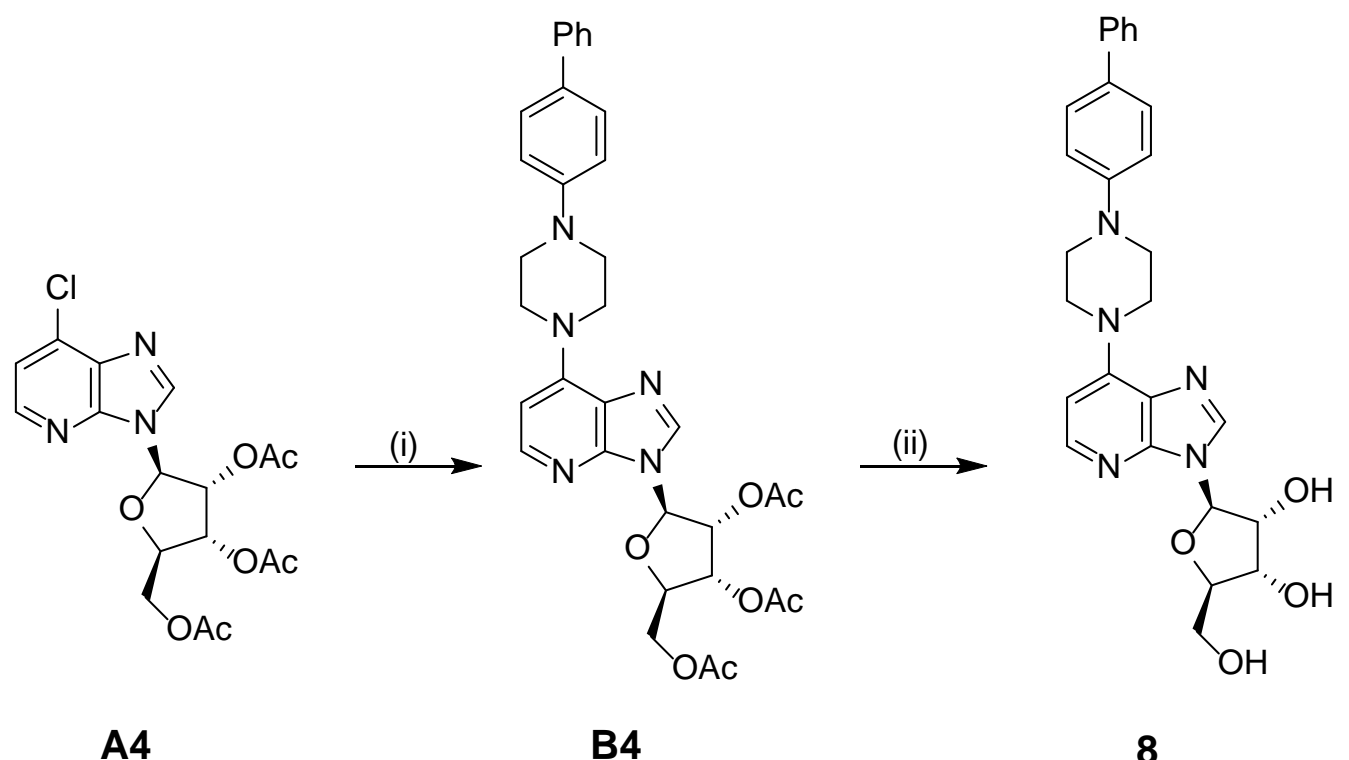

Reagents: (i) $\mathrm{Cs}_{2} \mathrm{CO}_{3}, 1$-([1,1'-biphenyl]-4-yl)piperazine, RuPhos Pd G2, tert-Amyl-OH, $100{ }^{\circ} \mathrm{C}$, $17 \mathrm{~h}$; (ii) $\mathrm{NH}_{4} \mathrm{OH}, \mathrm{MeOH}, 15^{\circ} \mathrm{C}, 24 \mathrm{~h}$.

(2R,3R,4R,5R)-2-(7-(4-([1,1'-biphenyl]-4-yl)piperazin-1-yl)-3H-imidazo[4,5-b]pyridin-3-yl)5-(acetoxymethyl)tetrahydrofuran-3,4-diyl diacetate (B4)

To the suspension of (2R,3R,4R,5R)-2-(acetoxymethyl)-5-(7-chloro-3H-imidazo[4,5b]pyridin-3-yl)tetrahydrofuran-3,4-diyl diacetate $\mathbf{A 4}$ (75 mg, 0.17 mmol) $)^{2}$, $\mathrm{Cs}_{2} \mathrm{CO}_{3}(166 \mathrm{mg}, 0.51$ mmol) and 1-([1,1'-biphenyl]-4-yl)piperazine (63 mg, $0.25 \mathrm{mmol}$ ) in tert-amyl alcohol (2 mL) was added RuPhos Pd G2 (13 mg, $0.017 \mathrm{mmol}$ ) and it was refluxed for $17 \mathrm{~h}$ under $\mathrm{N}_{2}$ atmosphere. After cooling to room temperature, $\mathrm{MeOH}(1 \mathrm{~mL})$ was added and it was filtered. The filtrate was concentrated under reduced pressure to afford crude (2R,3R,4R,5R)-2-(7-(4-([1,1'-biphenyl]-4yl)piperazin-1-yl)-3H-imidazo[4,5-b]pyridin-3-yl)-5-(acetoxymethyl)tetrahydrofuran-3,4-diyl diacetate B4 (133 mg, 49.6\% yield (38.8\% purity)) as a yellow solid which was directly used in the next reaction without further purification. MS (ESI) m/z: 488.1, 530.1[M-83\&M-125]+. 
Scheme C: Synthesis of 9, 10, 12, 13, 14, 16

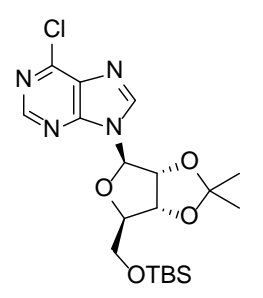

A2

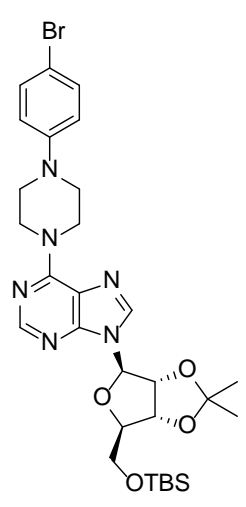

B2
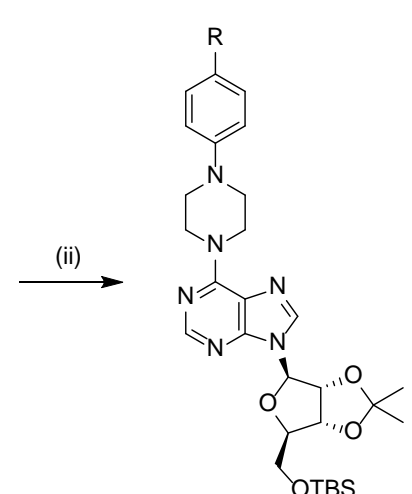

B3

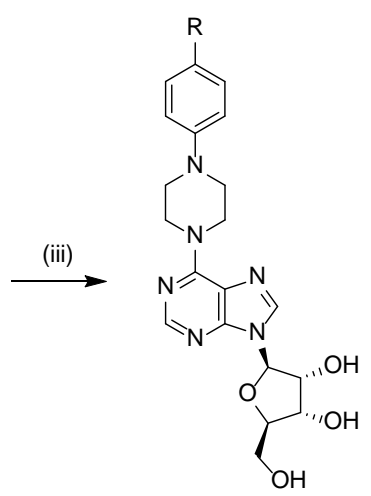

$9,10,12,13,14,16$

Reagents: (i) 1-(4-bromophenyl)piperazine, DIEA, EtOH, $80{ }^{\circ} \mathrm{C}, 17$ h; (ii) $\mathrm{RB}(\mathrm{OH})_{2}, \mathrm{~K}_{3} \mathrm{PO}_{4}$, XPhos Pd G2, THF/ $\mathrm{H}_{2} \mathrm{O}, 70{ }^{\circ} \mathrm{C}, 17 \mathrm{~h}$; (iii) TFA, THF/ $\mathrm{H}_{2} \mathrm{O}, 15-25^{\circ} \mathrm{C}, 2-17 \mathrm{~h}$.

6-(4-(4-bromophenyl)piperazin-1-yl)-9-((3aR,4R,6R,6aR)-6-((tert-

butyldimethylsilyl)oxy)methyl)-2,2-dimethyltetrahydrofuro[3,4-d][1,3]dioxol-4-yl)-9Hpurine (B2)

To a suspension of 1-(4-bromophenyl)piperazine (2.7 g, $8.84 \mathrm{mmol})$ in anhydrous EtOH (70 mL) were added DIEA (4.28 mL, $24.48 \mathrm{mmol})$ and 9-((3aR,4R,6R,6aR)-6-(((tertbutyldimethylsilyl)oxy)methyl)-2,2-dimethyltetrahydrofuro[3,4-d][1,3]dioxol-4-yl)-6-chloro-9Hpurine A2 (2.7 g, $6.12 \mathrm{mmol})^{25}$ at room temperature and it was stirred at $80{ }^{\circ} \mathrm{C}$ for $17 \mathrm{~h}$. The mixture was diluted with EtOH (30 mL), filtered. The resulting solid was washed with EtOH (20 $\mathrm{mL} \times 3$ ), dried under reduced pressure to afford 6-(4-(4-bromophenyl)piperazin-1-yl)-9((3aR,4R,6R,6aR)-6-(((tert-butyldimethylsilyl)oxy)methyl)-2,2-dimethyltetrahydrofuro[3,4-

d][1,3]dioxol-4-yl)-9H-purine B2 (3.5 g, 90\% yield) as a white solid. MS (ESI) m/z: 645.2, 647.2 $[\mathrm{M}+\mathrm{H}]^{+} \& 667.2,669.2[\mathrm{M}+\mathrm{Na}]^{+}$.

9-((3aR,4R,6R,6aR)-6-(((tert-butyldimethylsilyl)oxy)methyl)-2,2dimethyltetrahydrofuro[3,4-d][1,3]dioxol-4-yl)-6-(4-(4-(6-methoxypyridin-3yl)phenyl)piperazin-1-yl)-9H-purine (B3-1, R=(6-methoxypyridin-3-yl)phenyl)

Into the suspension of (6-methoxypyridin-3-yl)boronic acid (27.5 mg, $0.180 \mathrm{mmol})$, 6-(4(4-bromophenyl)piperazin-1-yl)-9-((3aR,4R,6R,6aR)-6-(((tert-butyldimethylsilyl)oxy)methyl)2,2-dimethyltetrahydrofuro[3,4-d][1,3]dioxol-4-yl)-9H-purine B2 (97 mg, $0.150 \mathrm{mmol}$ ), and 
XPhos Pd G2 (11.8 mg, $0.015 \mathrm{mmol})$ in anhydrous THF (1.5 mL) was added potassium phosphate tribasic $(0.300 \mathrm{~mL}, 0.300 \mathrm{mmol})$. The mixture was stirred at $70{ }^{\circ} \mathrm{C}$ under $\mathrm{N}_{2}$ for $17 \mathrm{~h}$ and then diluted with EtOAc (20 mL), washed with water $(10 \mathrm{~mL})$, separated, dried over $\mathrm{MgSO}_{4}$, filtered and concentrated to give the crude product 9-((3aR,4R,6R,6aR)-6-(((tertbutyldimethylsilyl)oxy)methyl)-2,2-dimethyltetrahydrofuro[3,4-d][1,3]dioxol-4-yl)-6-(4-(4-(6methoxypyridin-3-yl)phenyl)piperazin-1-yl)-9H-purine B3-1 (100 mg, 87\% yield) as a yellow solid, which was used in next step without further purification. MS (ESI) m/z: $674.3[\mathrm{M}+\mathrm{H}]^{+}$.

Scheme D: Synthesis of (2R,3S,4R,5R)-2-(hydroxymethyl)-5-(6-(4-(phenylethynyl)phenyl)9H-purin-9-yl)tetrahydrofuran-3,4-diol (11) and (2R,3R,4S,5R)-2-(6-([1,1'-biphenyl]-4ylethynyl)-9H-purin-9-yl)-5-(hydroxymethyl) tetrahydrofuran-3,4-diol (17)<smiles>CC1(C)O[C@@H]2[C@H](O1)[C@H](n1cnc3c(Cl)ncnc31)O[C@@H]2CO[Sb]</smiles>

A2

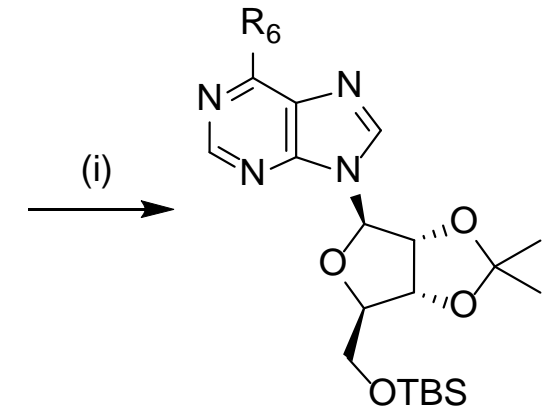

C1 (ii)<smiles>[R6]c1ncnc2c1ncn2[C@@H]1O[C@H](CO)[C@@H](O)[C@H]1O</smiles>

Reagents: (i) 4,4,5,5-tetramethyl-2-(4-(phenylethynyl)phenyl)-1,3,2-dioxaborolane, $\mathrm{K}_{3} \mathrm{PO}_{4}$, XPhos Pd G2, THF, $70{ }^{\circ} \mathrm{C}, 17$ h or 4-ethynyl-1,1'-biphenyl, $\mathrm{Cs}_{2} \mathrm{CO}_{3}$, CuI, XPhos Pd G2, $\mathrm{CH}_{3} \mathrm{CN}$, $90{ }^{\circ} \mathrm{C}, 17 \mathrm{~h}$; (ii) TFA, THF/ $\mathrm{H}_{2} \mathrm{O}, 20^{\circ} \mathrm{C}, 17 \mathrm{~h}$.

9-((3aR,4R,6R,6aR)-6-(((tert-butyldimethylsilyl)oxy)methyl)-2,2-

dimethyltetrahydrofuro[3,4-d][1,3]dioxol-4-yl)-6-(4-(phenylethynyl)phenyl)-9H-purine (C11, R6 $=4$-(phenylethynyl)phenyl)

To a suspension of 4,4,5,5-tetramethyl-2-(4-(phenylethynyl)phenyl)-1,3,2-dioxaborolane (260 mg, $0.49 \mathrm{mmol}), \quad$ 9-((3aR,4R,6R,6aR)-6-((tert-butyldimethylsilyl)oxy)methyl)-2,2dimethyltetrahydrofuro[3,4-d][1,3]dioxol-4-yl)-6-chloro-9H-purine A2 (100 mg, $0.23 \mathrm{mmol})^{25}$ in 
aqueous $\mathrm{K}_{3} \mathrm{PO}_{4}(1 \mathrm{M})$ and THF (2.0 mL) was added XPhos Pd G2 (9 mg, $0.000011 \mathrm{mmol}$ ) under $\mathrm{N}_{2}$ atmosphere and it was stirred at $70{ }^{\circ} \mathrm{C}$ for $17 \mathrm{~h}$. The mixture was diluted with water (20 mL), extracted with EtOAc (10 mLx3). The combined organic layers were washed with brine (20 mLx3), dried over anhydrous $\mathrm{Na}_{2} \mathrm{SO}_{4}$ and filtered. The filtrate was concentrated under reduced pressure to give crude 9-((3aR,4R,6R,6aR)-6-((tert-butyldimethylsilyl)oxy)methyl)-2,2dimethyltetrahydrofuro[3,4-d][1,3]dioxol-4-yl)-6-(4-(phenylethynyl)phenyl)-9H-purine C1-1 (260 mg, 95\% yield) as a yellow oil which was directly used in the next reaction without further purification. MS (ESI) m/z: 583.1[M+H] .

\section{6-([1,1'-biphenyl]-4-ylethynyl)-9-((3aR,4R,6R,6aR)-6-((tert-}

\section{butyldimethylsilyl)oxy)methyl)-2,2-dimethyltetrahydrofuro[3,4-d][1,3]dioxol-4-yl)-9H- purine (C1-2, $\mathbf{R}_{6}=[1,1$ '-biphenyl]-4-ylethynyl)}

To the suspension of 4-ethynyl-1,1'-biphenyl (58 mg, $0.32 \mathrm{mmol}$ ), $\mathrm{Cs}_{2} \mathrm{CO}_{3}$ (163 mg, 0.50 $\mathrm{mmol})$, CuI $(0.950 \mathrm{mg}, 4.99 \mu \mathrm{mol})$ and the XPhos Pd G2 (10 mg, $0.012 \mathrm{mmol})$ in anhydrous $\mathrm{CH}_{3} \mathrm{CN}(1 \mathrm{~mL})$ was added 9-((3aR,4R,6R,6aR)-6-((tert-butyldimethylsilyl)oxy)methyl)-2,2dimethyltetrahydrofuro[3,4-d][1,3]dioxol-4-yl)-6-chloro-9H-purine A2 (110 mg, 0.25 mmol) ${ }^{25}$ under $\mathrm{N}_{2}$ atmosphere. After it was stirred at $90{ }^{\circ} \mathrm{C}$ for $17 \mathrm{~h}$ under $\mathrm{N}_{2}$ atmosphere, the mixture was concentrated and purified by PTLC $\left(\mathrm{SiO}_{2}\right.$, Petroleum ether : EtOAc $\left.=3: 1\right)$ to give 6-([1,1'biphenyl]-4-ylethynyl)-9-((3aR,4R,6R,6aR)-6-(((tert-butyldimethylsilyl)oxy)methyl)-2,2dimethyltetrahydrofuro[3,4-d][1,3]dioxol-4-yl)-9H-purine C1-2 (46 mg, 27.2\% yield) as a yellow solid. MS (ESI) m/z: $583.1[\mathrm{M}+\mathrm{H}]^{+}$. 
Scheme F: Synthesis of (1R,2S,3R,5R)-3-(6-(4-([1,1'-biphenyl]-4-yl)piperazin-1-yl)-9Hpurin-9-yl)-5-(hydroxymethyl)cyclopentane-1,2-diol (15)<smiles>OC[C@H]1C[C@@H](n2cnc3c(Cl)ncnc32)[C@H](O)[C@@H]1O</smiles>

A3

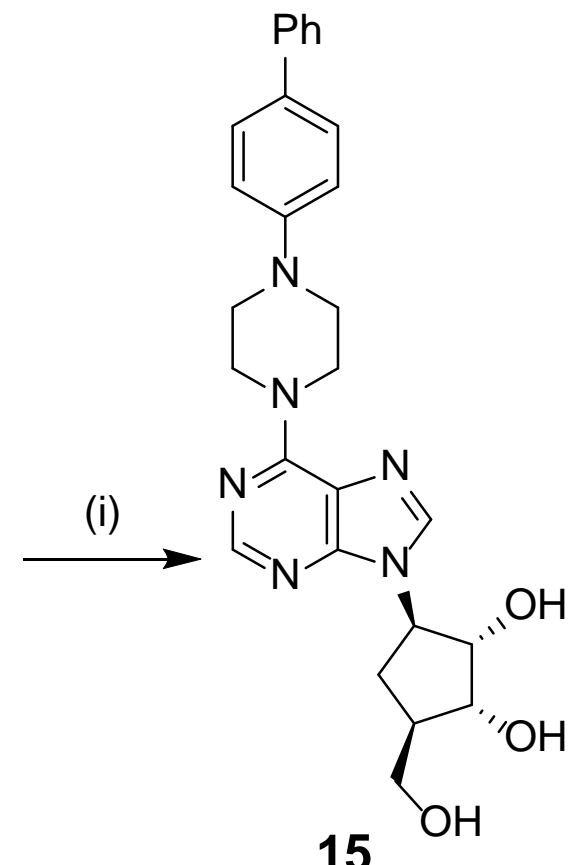

15

Reagents: (i) 1-([1,1'-biphenyl]-4-yl)piperazine, DIEA, EtOH, $70^{\circ} \mathrm{C}, 17 \mathrm{~h}$.

\section{Synthesis of 19-24}

Scheme 1: Synthesis of N-((2R,3S,4R,5R)-3,4-dihydroxy-5-(6-(4-(4-

(trifluoromethyl)phenyl)piperazin-1-yl)-9H-purin-9-yl)tetrahydrofuran-2-yl)methyl)-4methylbenzenesulfonamide (19)

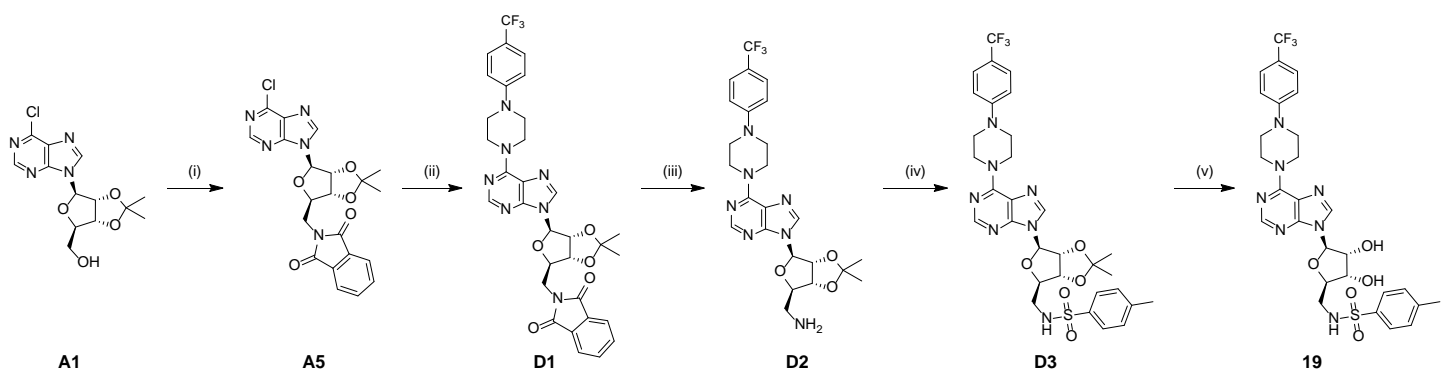

Reagents: (i) Phthalimide, DIAD, $\quad \mathrm{PPh}_{3}, \quad \mathrm{THF}, \quad 25 \quad{ }^{\circ} \mathrm{C}, \quad 17 \quad \mathrm{~h} ; \quad$ (ii) 1-(4(trifluoromethyl)phenyl)piperazine, DIEA, EtOH, $60^{\circ} \mathrm{C}, 17 \mathrm{~h}$; (iii) hydrazine hydrate, EtOH, $90{ }^{\circ} \mathrm{C}, 17 \mathrm{~h}$; (iv) 4-methylbenzenesulfonyl chloride, DIEA, DCM, $15{ }^{\circ} \mathrm{C}, 17 \mathrm{~h}$; (v) TFA, $\mathrm{THF} / \mathrm{H}_{2} \mathrm{O}, 15^{\circ} \mathrm{C}, 17 \mathrm{~h}$. 


\section{2-((3aR,4R,6R,6aR)-6-(6-chloro-9H-purin-9-yl)-2,2-dimethyltetrahydrofuro[3,4-}

\section{d][1,3]dioxol-4-yl)methyl)isoindoline-1,3-dione (A5)}

The mixture of triphenylphosphine (482 mg, 1.836 mmol), ((3aR,4R,6R,6aR)-6-(6-chloro9H-purin-9-yl)-2,2-dimethyltetrahydrofuro[3,4-d][1,3]dioxol-4-yl)methanol A1 (400 mg, 1.224 mmol), phthalimide (189 mg, $1.285 \mathrm{mmol})$ in THF (10 mL) and DIAD (0.286 mL, $1.469 \mathrm{mmol})$ was stirred at $25^{\circ} \mathrm{C}$ for $17 \mathrm{~h}$ then concentrated and purified by silica gel column chromatography (Petroleum ether : EtOAc =1 : 1) to give 2-(((3aR,4R,6R,6aR)-6-(6-chloro-9H-purin-9-yl)-2,2dimethyltetrahydrofuro[3,4-d][1,3]dioxol-4-yl)methyl)isoindoline-1,3-dione A5 (600 mg, 95\% yield) as a white solid. MS (ESI) m/z: $456.1[\mathrm{M}+\mathrm{H}]^{+}$.

\section{2-((3aR,4R,6R,6aR)-2,2-dimethyl-6-(6-(4-(4-(trifluoromethyl)phenyl)piperazin-1-yl)-9H-} purin-9-yl)tetrahydrofuro[3,4-d][1,3]dioxol-4-yl)methyl)isoindoline-1,3-dione (D1)

To the mixture of DIEA (0.607 $\mathrm{mL}, 3.47 \quad \mathrm{mmol})$ and 1-(4(trifluoromethyl)phenyl)piperazine (267 mg, $1.158 \mathrm{mmol}$ ) in EtOH $(5 \mathrm{~mL})$ was added 2(((3aR,4R,6R,6aR)-6-(6-chloro-9H-purin-9-yl)-2,2-dimethyltetrahydrofuro[3,4-d][1,3]dioxol-4yl)methyl)isoindoline-1,3-dione A5 (600 mg, $1.158 \mathrm{mmol}$ ). Then the mixture was stirred at $60^{\circ} \mathrm{C}$ for $17 \mathrm{~h}$, cooled, filtered to give 2-(((3aR,4R,6R,6aR)-2,2-dimethyl-6-(6-(4-(4(trifluoromethyl)phenyl)piperazin-1-yl)-9H-purin-9-yl)tetrahydrofuro[3,4-d][1,3]dioxol-4yl)methyl)isoindoline-1,3-dione D1 (650 mg, 82\% yield) as yellow solid. ${ }^{1} \mathrm{H}$ NMR ( $\mathrm{CD}_{3} \mathrm{OD}, 400$ MHz) $\delta 8.13$ (s, 3H), 7.77-7.76 (m, 3H), 7.67 (dd, $\left.J_{1}=1.6 \mathrm{~Hz}, J_{2}=4.8 \mathrm{~Hz}, 2 \mathrm{H}\right), 7.49$ (d, $J=8.4$ Hz, 2H), 6.95 (d, $J=8.4$ Hz, 2H), 6.02 (s, 1H), 6.22-6.10 (m, 1H), 5.50 (dd, $J_{1}=3.2 \mathrm{~Hz}, J_{2}=6.4$ $\mathrm{Hz}, 1 \mathrm{H}$ ), 5.22 (dd, $J_{1}=3.6 \mathrm{~Hz}, J_{2}=6.4 \mathrm{~Hz}, 1 \mathrm{H}$ ), 4.55-4.43 (m, 5H), 3.95-4.05 (m, 2H), 3.39 (brs, 4H), 1.56 (s, 3H), 1.33 (s, 3H). MS (ESI) m/z: $650.1[\mathrm{M}+\mathrm{H}]^{+}$.

((3aR,4R,6R,6aR)-2,2-dimethyl-6-(6-(4-(4-(trifluoromethyl)phenyl)piperazin-1-yl)-9Hpurin-9-yl)tetrahydrofuro[3,4-d][1,3]dioxol-4-yl)methanamine (D2).

The mixture of 2-(((3aR,4R,6R,6aR)-2,2-dimethyl-6-(6-(4-(4(trifluoromethyl)phenyl)piperazin-1-yl)-9H-purin-9- $\quad$ yl)tetrahydrofuro[3,4-d][1,3]dioxol-4yl)methyl)isoindoline-1,3-dione D1 (650 mg, $1.0 \mathrm{mmol}$ ) and hydrazine hydrate (102 mg, 2.0 $\mathrm{mmol}$ ) in $\mathrm{EtOH}(5 \mathrm{~mL})$ was stirred at $90^{\circ} \mathrm{C}$ for $17 \mathrm{~h}$, then it was cooled, filtered, and concentrated to give ((3aR,4R,6R,6aR)-2,2-dimethyl-6-(6-(4-(4-(trifluoromethyl)phenyl)piperazin-1-yl)-9H- 
purin-9-yl)tetrahydrofuro[3,4-d][1,3]dioxol-4-yl)methanamine F4 (450 $\mathrm{mg}, 78 \%$ yield) as a yellow solid, which was used in next step without further purification. ${ }^{1} \mathrm{H}$ NMR $\left(\mathrm{CD}_{3} \mathrm{OD}, 400\right.$ MHz) $\delta$ 8.33-8.18 (m, 2H), 7.50 (d, $J=8.6$ Hz, 2H), 7.17-7.02 (m, 2H), 6.22-6.10 (m, 1H), 5.45 (dd, $J_{1}=3.0 \mathrm{~Hz}, J_{2}=6.3 \mathrm{~Hz}, 1 \mathrm{H}$ ), $5.02\left(\mathrm{dd}, J_{1}=3.4 \mathrm{~Hz}, J_{2}=6.3 \mathrm{~Hz}, 1 \mathrm{H}\right.$ ), 4.43 (brs, $4 \mathrm{H}$ ), 4.304.19 (m, 1H), 3.52-3.38 (m, 2H), 3.00-2.81 (m, 1H), 1.60 (s, 3H), 1.38 (s, 3H). MS (ESI) m/z: $520.2[\mathrm{M}+\mathrm{H}]^{+}$.

\section{N-(((3aR,4R,6R,6aR)-2,2-dimethyl-6-(6-(4-(4-(trifluoromethyl)phenyl)piperazin-1-yl)-9H-} purin-9-yl)tetrahydrofuro[3,4-d][1,3]dioxol-4-yl)methyl)-4-methylbenzenesulfonamide (D3)

To a solution of $\quad$ ((3aR,4R,6R,6aR)-2,2-dimethyl-6-(6-(4-(4(trifluoromethyl)phenyl)piperazin-1-yl)-9H-purin-9-yl)tetrahydrofuro[3,4-d][1,3]dioxol-4yl)methanamine $\mathbf{D} 2(50 \mathrm{mg}, 0.096 \mathrm{mmol}$ ) and DIEA (0.050 mL, $0.289 \mathrm{mmol})$ in anhydrous DCM ( $2 \mathrm{~mL}$ ) was added 4-methylbenzene-1-sulfonyl chloride (18.35 mg, $0.096 \mathrm{mmol}$ ). The mixture was stirred at $15{ }^{\circ} \mathrm{C}$ for $17 \mathrm{~h}$, then it was concentrated under reduced pressure to afford $\mathrm{N}$ (((3aR,4R,6R,6aR)-2,2-dimethyl-6-(6-(4-(4-(trifluoromethyl)phenyl)piperazin-1-yl)-9H-purin-9yl)tetrahydrofuro[3,4-d][1,3]dioxol-4-yl)methyl)-4-methylbenzenesulfonamide D3 (72 mg, 94\% yield) as a light yellow oil. The crude product was used in next step without purification. MS (ESI) $\mathrm{m} / \mathrm{z}: 674.3[\mathrm{M}+\mathrm{H}]^{+}$. 
Scheme 2: Synthesis of (2R,3S,4R,5R)-2-(((4-nitrophenyl)amino)methyl)-5-(6-(4-(4-

(trifluoromethyl)phenyl)piperazin-1-yl)-9H-purin-9-yl)tetrahydrofuran-3,4-diol (20)

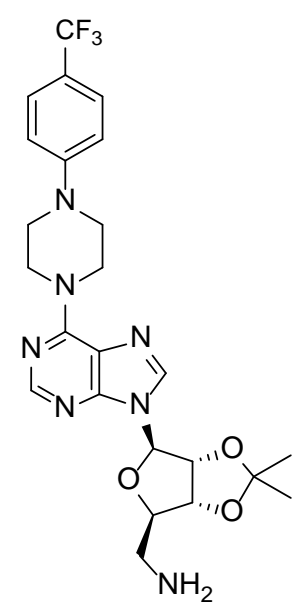

D2

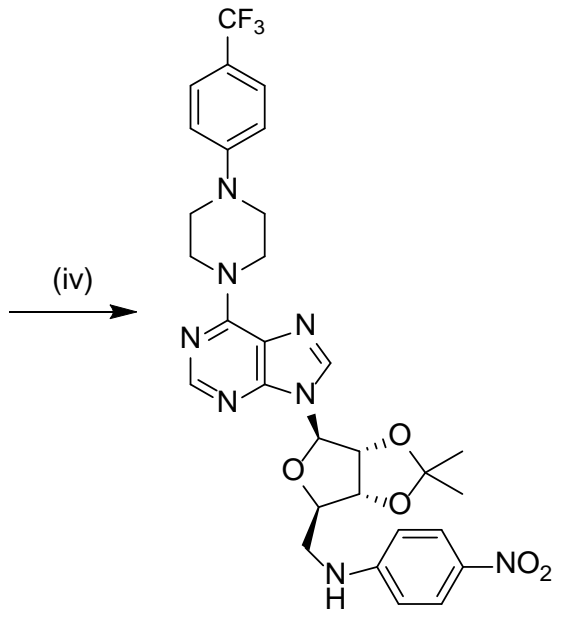

D4

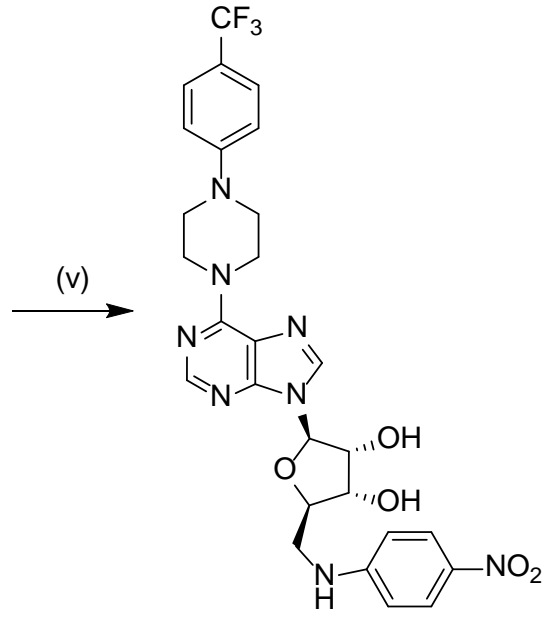

20

Reagents: (i) 1-fluoro-4-nitrobenzene, $\mathrm{K}_{2} \mathrm{CO}_{3}$, DABCO, DMF, $100{ }^{\circ} \mathrm{C}, 17 \mathrm{~h}$; (ii) TFA, $\mathrm{THF} / \mathrm{H}_{2} \mathrm{O}, 20^{\circ} \mathrm{C}, 17 \mathrm{~h}$.

Synthesis of N-((3aR,4R,6R,6aR)-2,2-dimethyl-6-(6-(4-(4-

(trifluoromethyl)phenyl)piperazin-1-yl)-9H-purin-9-yl)tetrahydrofuro[3,4-d][1,3]dioxol-4yl)methyl)-4-nitroaniline (D4)

To a solution of ((3aR,4R,6R,6aR)-2,2-dimethyl-6-(6-(4-(4(trifluoromethyl)phenyl)piperazin-1-yl)-9H-purin-9- yl)tetrahydrofuro[3,4-d][1,3]dioxol-4yl)methanamine D2 (200 mg, $0.385 \mathrm{mmol}$ ) and 1-fluoro-4-nitrobenzene (109 mg, $0.770 \mathrm{mmol}$ ) in DMF (4 mL) was added $\mathrm{K}_{2} \mathrm{CO}_{3}(106 \mathrm{mg}, 0.770 \mathrm{mmol})$ and DABCO (86 mg, $\left.0.770 \mathrm{mmol}\right)$. The mixture was stirred at $100{ }^{\circ} \mathrm{C}$ for $17 \mathrm{~h}$, then it was diluted with EtOAc (50 mL), washed with water $(30 \mathrm{~mL})$, the organic layer was separated and concentrated to give $\mathrm{N}-(((3 \mathrm{aR}, 4 \mathrm{R}, 6 \mathrm{R}, 6 \mathrm{aR})-$ 2,2-dimethyl-6-(6-(4-(4- (trifluoromethyl)phenyl)piperazin-1-yl)-9H-purin-9yl)tetrahydrofuro[3,4-d][1,3]dioxol-4-yl)methyl)-4-nitroaniline F5 (160 mg, 57.7\% yield) as a yellow solid, which was used in next step directly without further purification. MS (ESI) m/z: $641.1[\mathrm{M}+\mathrm{H}]^{+}$. 
Scheme 3: Synthesis of N-((2R,3S,4R,5R)-3,4-dihydroxy-5-(6-(4-(4-

(trifluoromethyl)phenyl)piperazin-1-yl)-9H-purin-9-yl)tetrahydrofuran-2-

yl)methyl)benzamide (21)
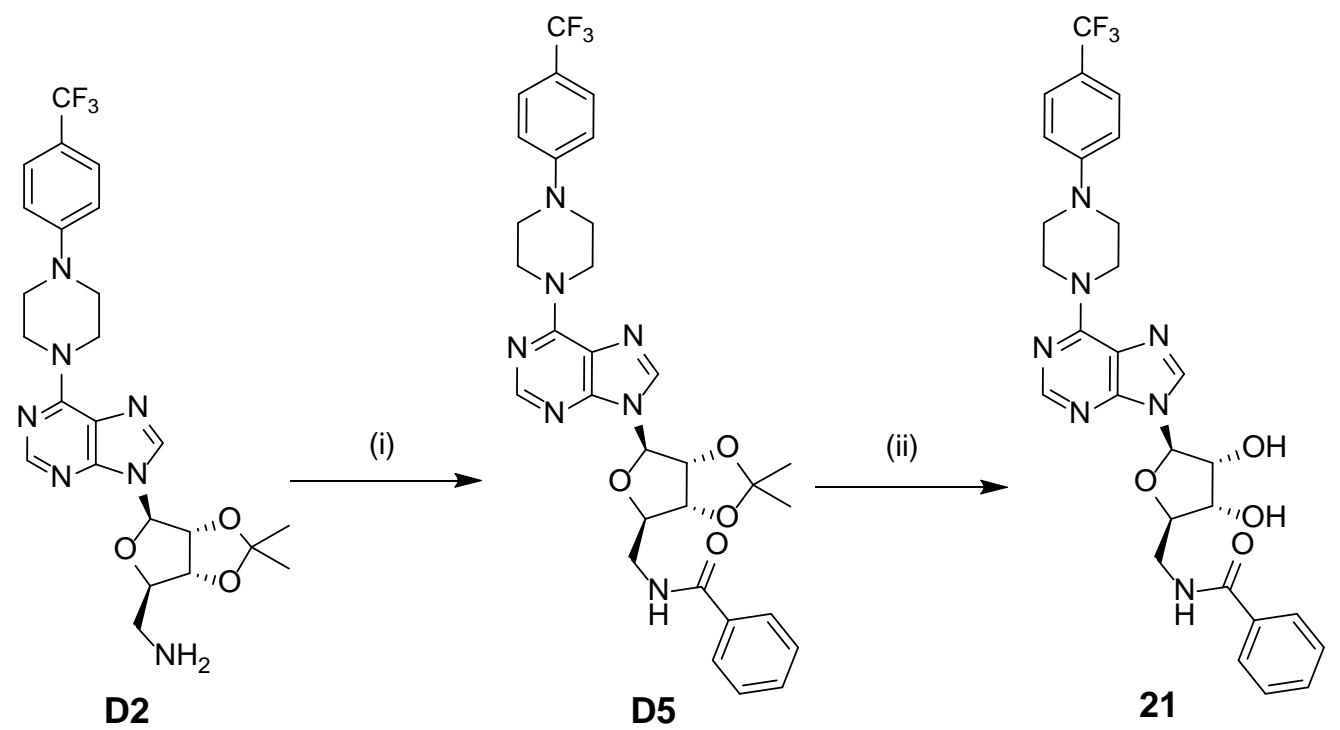

Reagents: (i) benzoyl chloride, DIEA, DCM, $15{ }^{\circ} \mathrm{C}, 17 \mathrm{~h}$; (ii) TFA, THF/ $\mathrm{H}_{2} \mathrm{O}, 15^{\circ} \mathrm{C}, 17 \mathrm{~h}$.

Synthesis of N-(((3aR,4R,6R,6aR)-2,2-dimethyl-6-(6-(4-(4-

(trifluoromethyl)phenyl)piperazin-1-yl)-9H-purin-9-yl)tetrahydrofuro[3,4-d][1,3]dioxol-4yl)methyl)benzamide (D5)

To a solution of $\quad$ ((3aR,4R,6R,6aR)-2,2-dimethyl-6-(6-(4-(4(trifluoromethyl)phenyl)piperazin-1-yl)-9H-purin-9-yl)tetrahydrofuro[3,4-d][1,3]dioxol-4yl)methanamine D2 (50 mg, $0.096 \mathrm{mmol}$ ) and DIEA (0.050 mL, $0.289 \mathrm{mmol})$ in anhydrous DCM ( $2 \mathrm{~mL}$ ) was added benzoyl chloride ( $16.23 \mathrm{mg}, 0.115 \mathrm{mmol})$. The mixture was stirred at $15^{\circ} \mathrm{C}$ for $17 \mathrm{~h}$, then it was concentrated under reduced pressure to afford $\mathrm{N}-(((3 a \mathrm{R}, 4 \mathrm{R}, 6 \mathrm{R}, 6 \mathrm{aR})-2,2-$ dimethyl-6-(6-(4-(4-(trifluoromethyl)phenyl)piperazin-1-yl)-9H-purin-9-yl)tetrahydrofuro[3,4d][1,3]dioxol-4-yl)methyl)benzamide D5 (68 mg, 94\% yield) as a light yellow oil. The crude product was used in next step without purification. MS (ESI) m/z: 624.3[M+H] $]^{+}$

Scheme 4: Synthesis of N-(4-(((2R,3S,4R,5R)-3,4-dihydroxy-5-(6-(4-(4(trifluoromethyl)phenyl)piperazin-1-yl)-9H-purin-9-yl)tetrahydrofuran-2yl)methyl)amino)phenyl)methanesulfonamide (22) 


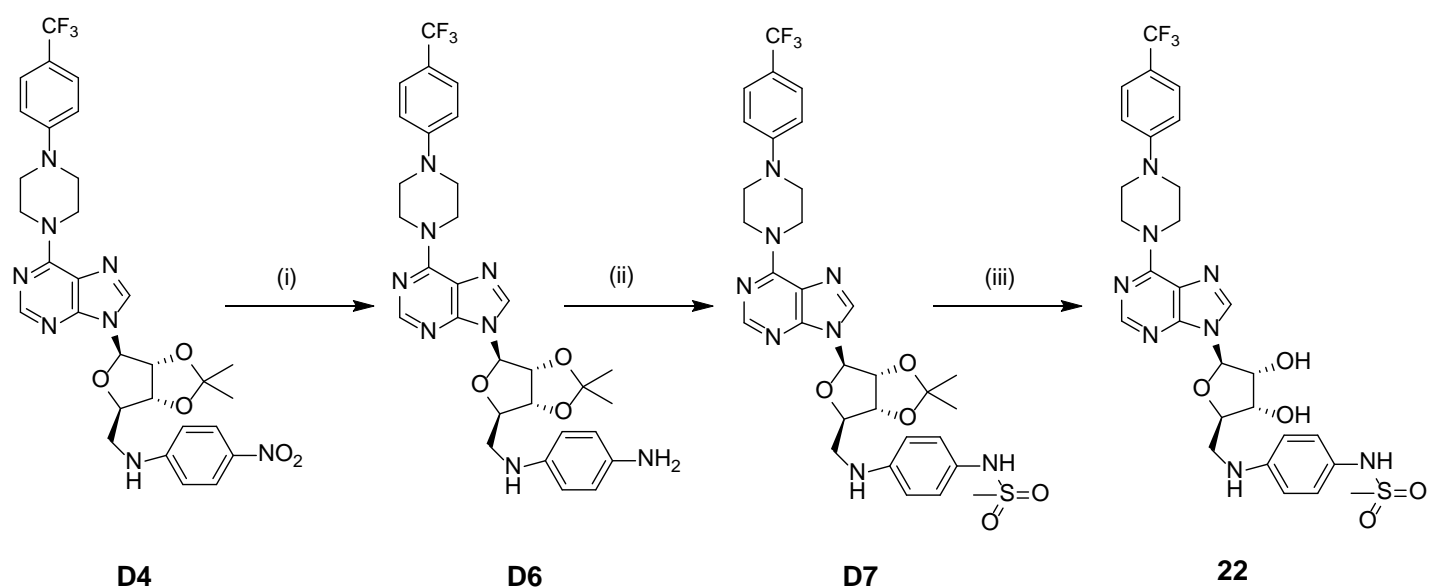

Reagents: (i) Pd/C, $\mathrm{H}_{2}$, EtOH, $20^{\circ} \mathrm{C}, 2$ h; (ii) MsCl, DIEA, DCM, $20^{\circ} \mathrm{C}, 17 \mathrm{~h}$; (iii) TFA, $\mathrm{THF} / \mathrm{H}_{2} \mathrm{O}, 20^{\circ} \mathrm{C}, 17 \mathrm{~h}$;

Synthesis of N1-((3aR,4R,6R,6aR)-2,2-dimethyl-6-(6-(4-(4(trifluoromethyl)phenyl)piperazin-1-yl)-9H-purin-9-yl)tetrahydrofuro[3,4-d][1,3]dioxol-4yl)methyl)benzene-1,4-diamine (D6)

The mixture of $\quad \mathrm{N}-(((3 \mathrm{aR}, 4 \mathrm{R}, 6 \mathrm{R}, 6 \mathrm{aR})-2,2-$ dimethyl-6-(6-(4-(4(trifluoromethyl)phenyl)piperazin-1-yl)-9H-purin-9- $\quad$ yl)tetrahydrofuro[3,4-d][1,3]dioxol-4yl)methyl)-4-nitroaniline D4 (100 mg, $0.156 \mathrm{mmol}$ ) and Pd/C (166 mg, $0.156 \mathrm{mmol})$ in EtOH (10 $\mathrm{mL}$ ) was stirred at $20^{\circ} \mathrm{C}$ for $2 \mathrm{~h}$ under $\mathrm{H}_{2}$ balloon. The mixture was filtered and the filtrate was concentrated to give N1-((3aR,4R,6R,6aR)-2,2-dimethyl-6-(6-(4-(4(trifluoromethyl)phenyl)piperazin-1-yl)-9H-purin-9-yl)tetrahydrofuro[3,4-d][1,3]dioxol-4yl)methyl)benzene-1,4-diamine D6 (100 mg, 86\% yield) as a yellow solid, which was used in next step directly. MS (ESI) m/z: $610.9[\mathrm{M}+\mathrm{H}]^{+}$.

Synthesis of N-(4-(((3aR,4R,6R,6aR)-2,2-dimethyl-6-(6-(4-(4(trifluoromethyl)phenyl)piperazin-1-yl)-9H-purin-9-yl)tetrahydrofuro[3,4-d][1,3]dioxol-4yl)methyl)amino)phenyl)methanesulfonamide (D7)

To the solution of DIEA $(0.051 \mathrm{~mL}, 0.295 \mathrm{mmol})$ and N1-(((3aR,4R,6R,6aR)-2,2dimethyl-6-(6-(4-(4- (trifluoromethyl)phenyl)piperazin- 
1-yl)-9H-purin-9-yl)tetrahydrofuro[3,4-d][1,3]dioxol-4-yl)methyl)benzene-1,4-diamine D6 (60 $\mathrm{mg}, 0.098 \mathrm{mmol}$ ) in DCM (5 mL) was added methanesulfonyl chloride (13.51 mg, $0.118 \mathrm{mmol}$ ) at $20^{\circ} \mathrm{C}$. The mixture was stirred at $20^{\circ} \mathrm{C}$ for $17 \mathrm{~h}$, then it was diluted with DCM $(20 \mathrm{~mL})$, washed with water $(10 \mathrm{~mL})$, separated, dried over $\mathrm{MgSO}_{4}$, filtered and concentrated to give $\mathrm{N}$-(4((((3aR,4R,6R,6aR)-2,2-dimethyl-6-(6-(4-(4-(trifluoromethyl)phenyl)piperazin-1-yl)-9H-purin9-yl)tetrahydrofuro[3,4-d][1,3]dioxol-4-yl)methyl)amino)phenyl)methanesulfonamide $\quad$ D7 (70 mg, 57.9\% yield) as a yellow solid. MS (ESI) m/z: $689.2[\mathrm{M}+\mathrm{H}]^{+}$.

Scheme 5: Synthesis of (2R,3R,4S,5R)-2-(6-(4-([1,1'-biphenyl]-4-yl)piperazin-1-yl)-9Hpurin-9-yl)-5-((benzyloxy)methyl)tetrahydrofuran-3,4-diol (23)

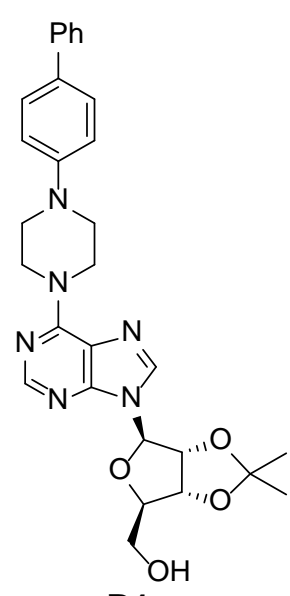

B1
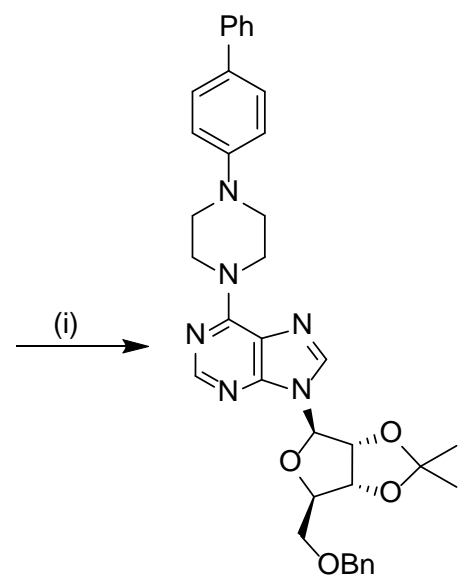

B5

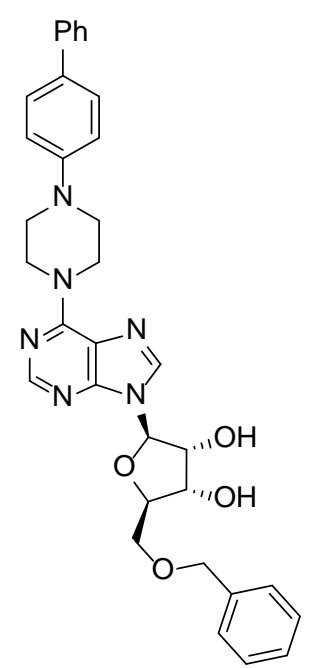

23

Reagents: (i) benzyl bromide, NaH, THF, $20^{\circ} \mathrm{C}, 17 \mathrm{~h}$; (ii) $\mathrm{HCl}$ in $\mathrm{MeOH}, 25^{\circ} \mathrm{C}, 1 \mathrm{~h}$.

Synthesis of 6-(4-([1,1'-biphenyl]-4-yl)piperazin-1-yl)-9-((3aR,4R,6R,6aR)-6-

((benzyloxy)methyl)-2,2-dimethyltetrahydrofuro[3,4-d][1,3]dioxol-4-yl)-9H-purine (B5)

To the solution of ((3aR,4R,6R,6aR)-6-(6-(4-([1,1'-biphenyl]-4-yl)piperazin-1-yl)-9Hpurin-9-yl)-2,2-dimethyltetrahydrofuro[3,4-d][1,3]dioxol-4-yl)methanol B1 (600 mg, $1.135 \mathrm{mmol}$ ) in THF ( $5 \mathrm{~mL}$ ) was added NaH (54.5 mg, $1.362 \mathrm{mmol}$ ) and then benzyl bromide (0.149 mL, 1.249 mmol) at $20^{\circ} \mathrm{C}$. The mixture was stirred at $20^{\circ} \mathrm{C}$ for $17 \mathrm{~h}$, then it was diluted with EtOAc (50 $\mathrm{mL})$, washed with water $(30 \mathrm{~mL})$ and brine $(30 \mathrm{~mL})$, separated, dried over $\mathrm{MgSO}_{4}$, and filtered, concentrated to give 6-(4-([1,1'-biphenyl]-4-yl)piperazin-1-yl)-9-((3aR,4R,6R,6aR)-6((benzyloxy)methyl)-2,2-dimethyltetrahydrofuro[3,4-d][1,3]dioxol-4-yl)-9H-purine B5 (700 mg, 
73.8\% yield) as a yellow solid. MS (ESI) m/z: $619.1[\mathrm{M}+\mathrm{H}]^{+}$.

Scheme 6: Synthesis of (2R,3S,4R,5R)-2-(((4-fluorophenyl)amino)methyl)-5-(6-(4-(4(trifluoromethyl)phenyl)piperazin-1-yl)-9H-purin-9-yl)tetrahydrofuran-3,4-diol (24)

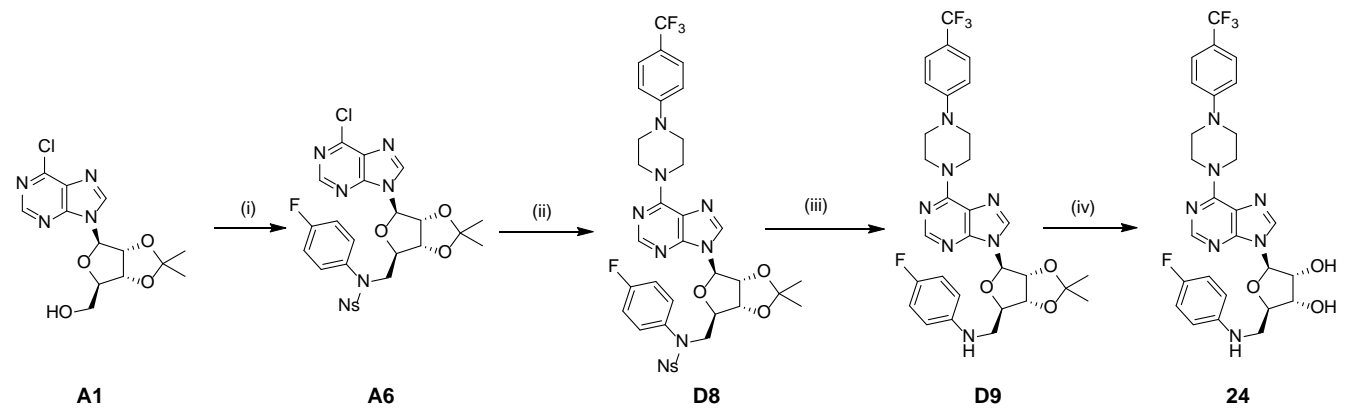

Reagents: (i) N-(4-fluorophenyl)-4-nitrobenzenesulfonamide, DIAD, PPh 3 , THF, $25{ }^{\circ} \mathrm{C}, 17 \mathrm{~h}$;

(iii) 1-(4-(trifluoromethyl)phenyl)piperazine, DIEA, EtOH, $70{ }^{\circ} \mathrm{C}, 17 \mathrm{~h}$; (iv) thiophenol, $\mathrm{K}_{2} \mathrm{CO}_{3}$, $\mathrm{CH}_{3} \mathrm{CN}, 40{ }^{\circ} \mathrm{C}, 17 \mathrm{~h}$; (v) $\mathrm{HCl}$ in $\mathrm{MeOH}, 25^{\circ} \mathrm{C}, 1 \mathrm{~h}$.

Synthesis of N-((3aR,4R,6R,6aR)-6-(6-chloro-9H-purin-9-yl)-2,2-

dimethyltetrahydrofuro[3,4-d][1,3]dioxol-4-yl)methyl)-N-(4-fluorophenyl)-4nitrobenzenesulfonamide (A6)

The mixture of triphenylphosphine (241 mg, 0.918 mmol), ((3aR,4R,6R,6aR)-6-(6-chloro9H-purin-9-yl)-2,2- dimethyltetrahydrofuro[3,4-d][1,3]dioxol-4-yl)methanol A1 (200 mg, 0.612 mmol) and N-(4-fluorophenyl)-2-nitrobenzenesulfonamide (181 mg, $0.612 \mathrm{mmol}$ ) and DIAD $(0.143 \mathrm{~mL}, 0.735 \mathrm{mmol})$ in THF $(10 \mathrm{~mL})$ was stirred at $25{ }^{\circ} \mathrm{C}$ for $17 \mathrm{~h}$, then it was concentrated and purified by silica gel column chromatography (Petroleum ether : EtOAc = 1:1) to give N(((3aR,4R,6R,6aR)-6-(6-chloro- 9H-purin-9-yl)-2,2-dimethyltetrahydrofuro[3,4-d][1,3]dioxol-4yl)methyl)-N-(4-fluorophenyl)-2-nitrobenzenesulfonamide A6 (300 mg, 64.8\% yield) as a white solid. ${ }^{1} \mathrm{H}$ NMR (400MHz, $\left.\mathrm{CDCl}_{3}\right) \delta 8.81$ (s, 1H), 8.15 (s, 1H), 7.57-7.50 (m, 2H), 7.40-7.38 (m, 2H), $7.08\left(\mathrm{dd}, J_{1}=3.6 \mathrm{~Hz}, J_{2}=8.8 \mathrm{~Hz}, 2 \mathrm{H}\right), 6.88(\mathrm{t}, J=8.4 \mathrm{~Hz}, 2 \mathrm{H}), 6.13(\mathrm{~s}, 1 \mathrm{H}), 5.61$ (d, $J=6.4$ $\mathrm{Hz}, 1 \mathrm{H}), 5.20$ (d, $J=4.4 \mathrm{~Hz}, 1 \mathrm{H}), 4.40-4.37$ (m, 1H), 4.16-4.14 (m, 1H), 3.92-3.83 (m, 1H), 1.58 (s, 3H), 1.41 (s, 3H). MS (ESI) m/z: $605.1[\mathrm{M}+\mathrm{H}]^{+}$.

Synthesis of N-((3aR,4R,6R,6aR)-2,2-dimethyl-6-(6-(4-(4(trifluoromethyl)phenyl)piperazin-1-yl)-9H-purin-9-yl)tetrahydrofuro[3,4-d][1,3]dioxol-4yl)methyl)-N-(4-fluorophenyl)-4-nitrobenzenesulfonamide (D8) 
To the solution of 1-(4-(trifluoromethyl)phenyl)piperazine (137 mg, $0.595 \mathrm{mmol})$ and DIEA (0.087 mL, 0.496 mmol) in EtOH (5 mL) was added N-(((3aR,4R,6R,6aR)-6-(6-chloro-9Hpurin-9-yl)-2,2-dimethyltetrahydrofuro[3,4-d][1,3]dioxol-4-yl)methyl)-N-(4-fluorophenyl)-2nitrobenzenesulfonamide A6 (300 mg, $0.496 \mathrm{mmol}$ ). The mixture was stirred at $70{ }^{\circ} \mathrm{C}$ for $17 \mathrm{~h}$, then it was cooled and concentrated, purified by silica gel column chromatography (Petroleum ether: $\quad \mathrm{EtOAc}=2$ : 1) to give $\mathrm{N}-(((3 \mathrm{aR}, 4 \mathrm{R}, 6 \mathrm{R}, 6 \mathrm{aR})-2,2-$ dimethyl-6-(6-(4-(4(trifluoromethyl)phenyl)piperazin-1-yl)-9H-purin-9-yl)tetrahydrofuro[3,4-d][1,3]dioxol-4yl)methyl)-N-(4-fluorophenyl)-2-nitrobenzenesulfonamide D8 (360 mg, 82\% yield) as a yellow solid. ${ }^{1} \mathrm{H} \mathrm{NMR}\left(\mathrm{CDCl}_{3}, 400 \mathrm{MHz}\right.$ ) $\delta 8.30$ (s, 1H), 7.72 (s, 1H), 7.55-7.51 (m, 4H), 7.38 (d, $J=2.8$ $\mathrm{Hz}, 2 \mathrm{H}), 7.02-6.97(\mathrm{~m}, 4 \mathrm{H}), 6.80$ (t, $J=7.6 \mathrm{~Hz}, 2 \mathrm{H}), 6.03(\mathrm{~s}, 1 \mathrm{H}), 6.73-6.62(\mathrm{~m}, 2 \mathrm{H}), 5.60$ (d, $J=$ $6.4 \mathrm{~Hz}, 1 \mathrm{H}), 5.12$ (d, $J=4.4 \mathrm{~Hz}, 1 \mathrm{H}), 4.46-4.40$ (m, 5H), 4.22-4.17 (m, 1H), 4.04-3.98 (m, 1H), 3.45-3.42 (m, 4H), 1.57 (s, 3H), 1.40 (s, 3H). MS (ESI) m/z: $798.9[\mathrm{M}+\mathrm{H}]^{+}$.

\section{Synthesis of N-((3aR,4R,6R,6aR)-2,2-dimethyl-6-(6-(4-(4-}

(trifluoromethyl)phenyl)piperazin-1-yl)-9H-purin-9-yl)tetrahydrofuro[3,4-d][1,3]dioxol-4yl)methyl)-4-fluoroaniline (D9)

The mixture of N-((3aR,4R,6R,6aR)-2,2-dimethyl-6-(6-(4-(4(trifluoromethyl)phenyl)piperazin-1-yl)-9H-purin- $\quad$ 9-yl)tetrahydrofuro[3,4-d][1,3]dioxol-4yl)methyl)-N-(4-fluorophenyl)- 2-nitrobenzenesulfonamide D8 (300 mg, 0.376 mmol), thiophenol (0.077 mL, $0.751 \mathrm{mmol}$ ) and $\mathrm{K}_{2} \mathrm{CO}_{3}$ (208 mg, $1.502 \mathrm{mmol}$ ) in $\mathrm{CH}_{3} \mathrm{CN}$ (2 mL) was stirred at 40 ${ }^{\circ} \mathrm{C}$ for $17 \mathrm{~h}$, then it was cooled, diluted with DCM (30 mL), washed with water $(30 \mathrm{~mL})$ and brine (30 mL), separated, dried over $\mathrm{MgSO}_{4}$, filtered and concentrated to give crude $\mathrm{N}$ (((3aR,4R,6R,6aR)-2,2-dimethyl-6-(6-(4-(4- (trifluoromethyl)phenyl)piperazin-1-yl)-9H-purin-9yl)tetrahydrofuro[3,4-d][1,3]dioxol-4-yl)methyl)-4-fluoroaniline D9 (200 mg, 60.7\% yield) as a yellow solid. MS (ESI) m/z: $614.3[\mathrm{M}+\mathrm{H}]^{+}$. 
a

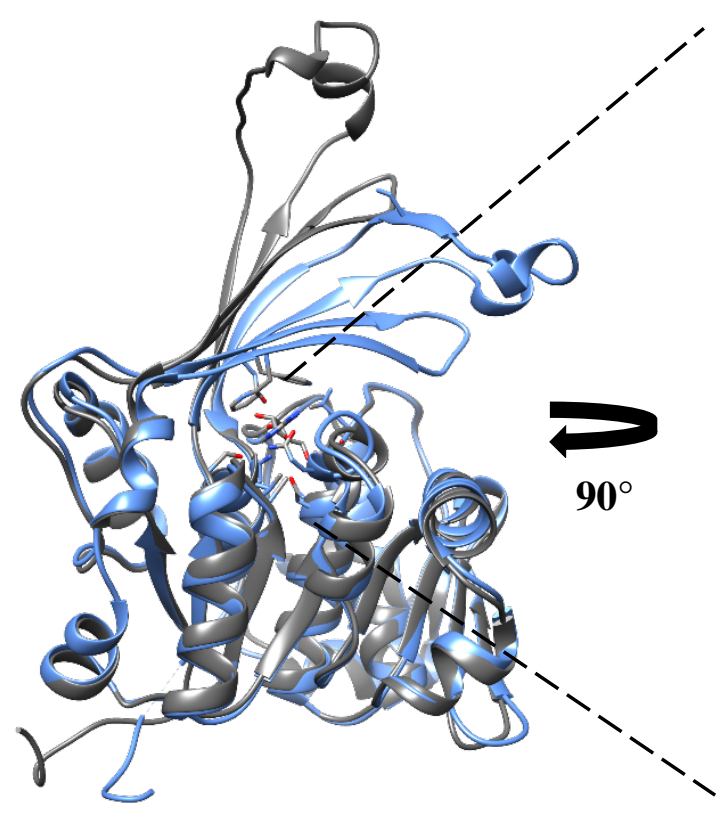

b

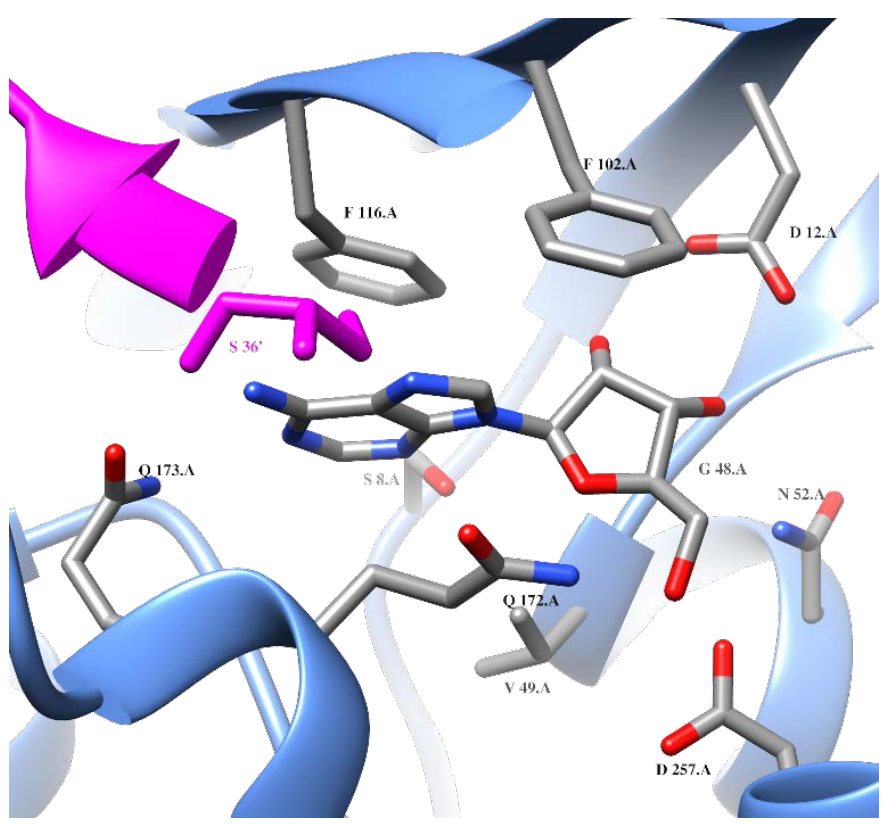

Figure S1. (a) Superimposition of the closed (blue, PDB ID 2PKM) and open conformations (gray, PDB ID 2PKF) of MtbAdoK. Only chain A of the dimer is shown. (b) Adenosine bound to the active site of MtbAdoK. Adenosine and residues involved in binding are shown as sticks and labeled with one-letter code and chain identifier. Chain B residues are colored magenta. 
Table S1. Close contacts of the MtbAdoK-2 complex $\leq 3.5 \AA$.

\begin{tabular}{|c|c|c|}
\hline Atom & Residue & Distance ( $(\AA)$ \\
\hline $\mathrm{C} 2$ & Ser8.A OG & 3.17 \\
\hline C2 & Ser8.A CB & 3.41 \\
\hline N3 & Ser8.A OG & 2.70 \\
\hline N3 & Ser8.A CB & 3.38 \\
\hline N6 & Gln173.A OE1 & 3.29 \\
\hline N6 & HOH501.B O & 3.36 \\
\hline $\mathrm{C} 2{ }^{\prime}$ & Asp12.A OD1 & 3.45 \\
\hline C3' & Asp12.A OD2 & 3.22 \\
\hline C5' & Asp257.A OD2 & 3.32 \\
\hline C5' & HOH618.A & 3.35 \\
\hline $\mathrm{O} 2{ }^{\prime}$ & Asp12.A OD1 & 2.67 \\
\hline $\mathrm{O} 2{ }^{\prime}$ & Gly48.A N & 3.08 \\
\hline $\mathrm{O}^{\prime}$ & Phe102.A CB & 3.35 \\
\hline $\mathrm{O}^{\prime}$ & Ala10.A CB & 3.48 \\
\hline O3' & Asp12.A OD2 & 2.53 \\
\hline O3' & Gly48.A N & 2.85 \\
\hline O3' & Asn52.A ND2 & 3.16 \\
\hline O3' & Asp12.A CG & 3.24 \\
\hline O3' & Asp12.A OD1 & 3.20 \\
\hline O3' & Gly47.A CA & 3.43 \\
\hline O4' & Val49.A CG1 & 3.31 \\
\hline O5' & Asp257.A OD2 & 2.65 \\
\hline O5' & HOH553.A & 2.72 \\
\hline O5' & Asp257.A CG & 3.41 \\
\hline IAE & HOH501.B O & 2.94 \\
\hline IAE & Ser36.B OG & 3.07 \\
\hline
\end{tabular}


Table S2. Crystal data collection and refinement statistics for MtbAdoK-2.

\begin{tabular}{|c|c|}
\hline Statistic & MtbAdoK-2 \\
\hline \multicolumn{2}{|c|}{ Data collection } \\
\hline Space Group & $\mathrm{P} 4_{1}$ \\
\hline \multicolumn{2}{|c|}{ Cell Dimensions } \\
\hline a, b, c $(\AA)$ & $49.0,49.0,262.2$ \\
\hline$\alpha, \beta, \gamma\left({ }^{\circ}\right)$ & $90,90,90$ \\
\hline Resolution $(\AA)$ & $34.6-2.1(2.18-2.10)$ \\
\hline $\mathrm{R}_{\text {merge }}$ & $0.07(0.24)$ \\
\hline $\mathrm{I} / \sigma \mathrm{I}$ & $23.3(7.63)$ \\
\hline Completeness \% & $96.5(98.49)$ \\
\hline Redundancy & 7.3 \\
\hline \multicolumn{2}{|c|}{ Refinement } \\
\hline Resolution $(\AA)$ & 2.1 \\
\hline No. of reflections & 34593 \\
\hline $\mathrm{R}_{\text {work }} / \mathrm{R}_{\text {free }}$ & $0.19 / 0.22$ \\
\hline \multicolumn{2}{|c|}{ No. of atoms } \\
\hline Protein & 4676 \\
\hline Ligand & 98 \\
\hline Water & 259 \\
\hline \multicolumn{2}{|c|}{ B factors } \\
\hline Protein & 50.6 \\
\hline Ligand/ion & 51.0 \\
\hline Water & 50.7 \\
\hline \multicolumn{2}{|c|}{ rmsd } \\
\hline Bond lengths ( $\AA$ ) & 0.004 \\
\hline Bond angles $\left({ }^{\circ}\right)$ & 1.01 \\
\hline
\end{tabular}


Table S3. Close contacts of the MtbAdoK-3 complex $\leq 3.5 \AA$.

\begin{tabular}{|c|c|c|}
\hline Atom & Residue & Distance ( $\AA$ ) \\
\hline C2 & Ser8.A CB & 3.44 \\
\hline C2 & Ser8.A OG & 3.30 \\
\hline N3 & Ser8.A OG & 2.76 \\
\hline N3 & Ser8.A CB & 3.35 \\
\hline N3 & Phe116.A CZ & 3.48 \\
\hline C4 & Phe116.A CE1 & 3.32 \\
\hline N6 & Gln173.A OE1 & 3.25 \\
\hline C8 & Phe102.A CD1 & 3.47 \\
\hline N9 & Phe116.A CE1 & 3.50 \\
\hline N11 & Ser36.B OG & 3.00 \\
\hline N11 & Phe102.A CE1 & 3.33 \\
\hline N11 & HOH601.A O & 3.31 \\
\hline $\mathrm{C}^{\prime}$ & Asp12.A OD1 & 3.46 \\
\hline C3' & Asp12.A OD2 & 3.21 \\
\hline C5' & Asp257.A OD2 & 3.33 \\
\hline C5' & HOH583.А O & 3.43 \\
\hline $\mathrm{O} 2{ }^{\prime}$ & Asp12.A OD1 & 2.70 \\
\hline $\mathrm{O} 2{ }^{\prime}$ & Gly48.A N & 3.01 \\
\hline $\mathrm{O}^{\prime}$ & Gly48.A CA & 3.41 \\
\hline O3' & Asp12.A OD2 & 2.39 \\
\hline O3' & Asp12.A CG & 3.17 \\
\hline O3' & Gly48.A N & 3.02 \\
\hline O3' & Asn52.A ND2 & 3.23 \\
\hline O3' & Asp12.A OD1 & 3.20 \\
\hline O4' & Val49.A CG1 & 3.43 \\
\hline O4' & Gln172.A NE2 & 3.23 \\
\hline O5' & Asp257.A OD2 & 2.60 \\
\hline O5' & Gln172.A NE2 & 2.83 \\
\hline O5' & HOH552.A O & 2.79 \\
\hline O5' & Asp257.A CG & 3.39 \\
\hline
\end{tabular}


Table S4. Crystal data collection and refinement statistics for MtbAdoK-3.

\begin{tabular}{|c|c|}
\hline Statistic & MtbAdoK-3 \\
\hline \multicolumn{2}{|c|}{ Data collection } \\
\hline Space Group & $\mathrm{P} 4_{1}$ \\
\hline \multicolumn{2}{|c|}{ Cell Dimensions } \\
\hline a, b, c $(\AA)$ & $48.9,48.9,262.0$ \\
\hline$\alpha, \beta, \gamma\left({ }^{\circ}\right)$ & $90,90,90$ \\
\hline Resolution $(\AA)$ & $34.5-2.1(2.17-2.10)$ \\
\hline $\mathrm{R}_{\text {merge }}$ & $0.07(0.24)$ \\
\hline $\mathrm{I} / \sigma \mathrm{I}$ & $22.8(6.85)$ \\
\hline Completeness \% & $98.2(99.80)$ \\
\hline Redundancy & 7.3 \\
\hline \multicolumn{2}{|c|}{ Refinement } \\
\hline Resolution $(\AA)$ & 2.1 \\
\hline No. of reflections & 35089 \\
\hline $\mathrm{R}_{\text {work }} / \mathrm{R}_{\text {free }}$ & $0.18 / 0.23$ \\
\hline \multicolumn{2}{|c|}{ No. of atoms } \\
\hline Protein & 4660 \\
\hline Ligand & 88 \\
\hline Water & 241 \\
\hline \multicolumn{2}{|c|}{ B factors } \\
\hline Protein & 46.5 \\
\hline Ligand/ion & 41.5 \\
\hline Water & 42.3 \\
\hline \multicolumn{2}{|c|}{ rmsd } \\
\hline Bond lengths ( $\AA$ ) & 0.01 \\
\hline Bond angles $\left({ }^{\circ}\right)$ & 1.09 \\
\hline
\end{tabular}



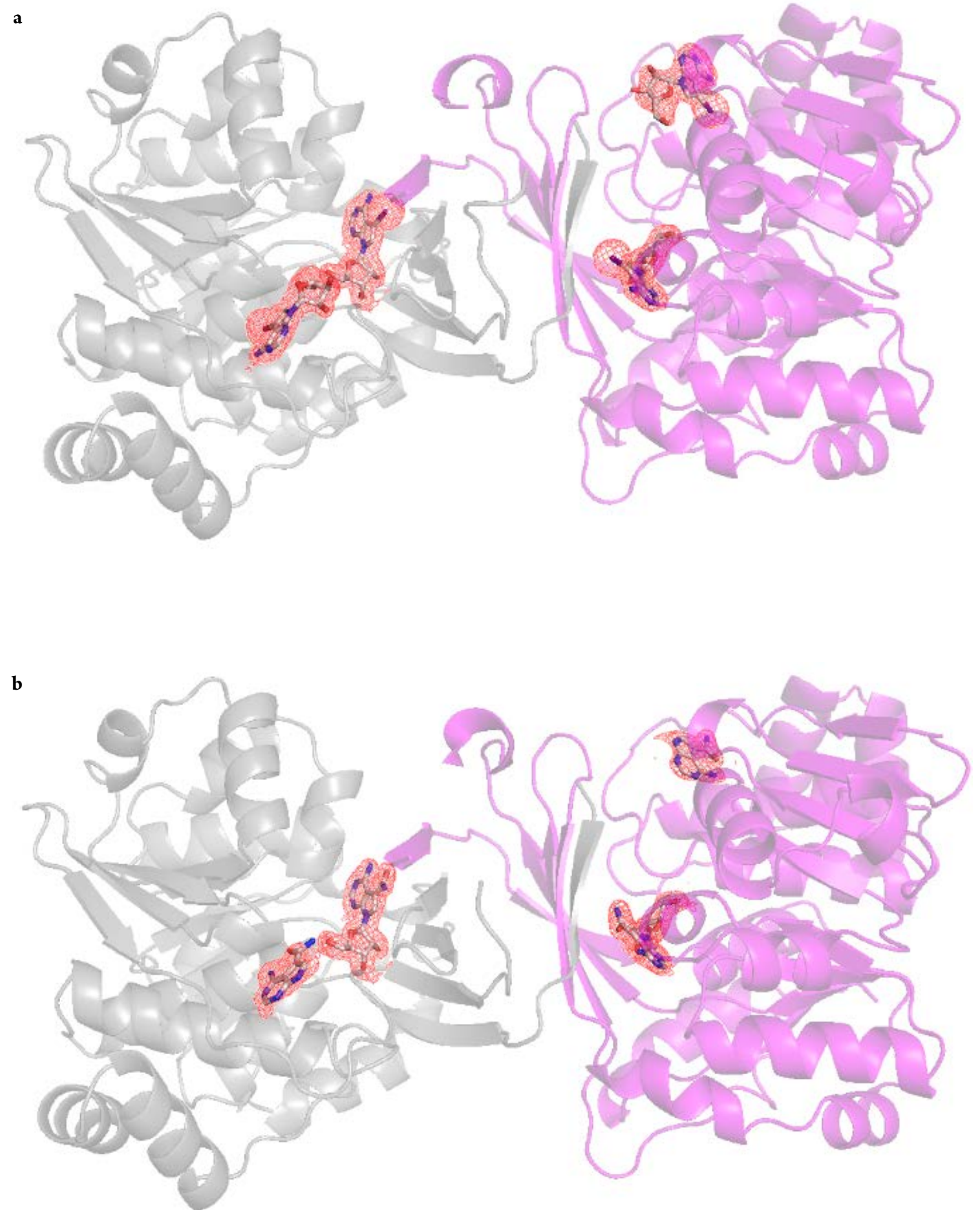

Figure S2. MtbAdoK homodimer embedded with two copies of compound 2 (a) and compound 3 (b) per chain. Chain $\mathrm{A}$ is colored gray, and chain $\mathrm{B}$ is colored magenta. $2 \mathrm{~F}_{\mathrm{o}}-\mathrm{F}_{\mathrm{c}}$ maps contoured at $1.6 \sigma$. 
Table S5. Close contacts of the MtbAdoK-1 complex $\leq 3.5 \AA$. Analyzed from PDB ID 2PKM.

\begin{tabular}{|c|c|c|}
\hline Atom & Residue & Distance ( $(\AA)$ \\
\hline C2 & Ser8.A OG & 3.11 \\
\hline N3 & Ser8.A OG & 2.62 \\
\hline N3 & Ser8.A CB & 3.47 \\
\hline N3 & Phe116.A CE1 & 3.42 \\
\hline $\mathrm{C} 4$ & Phe116.A CE1 & 3.38 \\
\hline $\mathrm{C} 4$ & Phe116.A CD1 & 3.39 \\
\hline C5 & Gln172.A CB & 3.46 \\
\hline C5 & Phe116.A CD1 & 3.39 \\
\hline N1 & Gln173.A NE2 & 2.99 \\
\hline N7 & Ser36.B OG & 2.65 \\
\hline C8 & Phe102.A CE1 & 3.27 \\
\hline $\mathrm{C} 8$ & Phe102.A CD1 & 3.48 \\
\hline C3' & Asp12.A OD2 & 3.35 \\
\hline C4' & Gln172.A NE2 & 3.49 \\
\hline $\mathrm{O} 4{ }^{\prime}$ & Val49.A CG1 & 3.22 \\
\hline O4' & Gln172.A NE2 & 3.26 \\
\hline C5' & Asp257.A OD2 & 2.87 \\
\hline C5' & Asn52.A ND2 & 3.31 \\
\hline O5' & Gln172.A NE2 & 2.62 \\
\hline O5' & Asp257.A OD2 & 2.69 \\
\hline O5' & Asp257.A CG & 3.32 \\
\hline O3' & Asp12.A OD2 & 2.70 \\
\hline O3' & Gly48.A N & 2.99 \\
\hline O3' & Asn52.A ND2 & 3.01 \\
\hline O3' & Asp12.A CG & 3.41 \\
\hline $\mathrm{O} 2{ }^{\prime}$ & Gly48.A N & 2.82 \\
\hline $\mathrm{O} 2{ }^{\prime}$ & Asp12.A OD1 & 2.76 \\
\hline $\mathrm{O} 2^{\prime}$ & Gly48.A CA & 3.28 \\
\hline N6 & Gln173.A OE1 & 2.96 \\
\hline N6 & Gln172.A O & 3.26 \\
\hline N6 & Ser36.B OG & 3.43 \\
\hline
\end{tabular}


Table S6. Close contacts of the MtbAdoK-4 complex $\leq 3.5 \AA$.

\begin{tabular}{|c|c|c|}
\hline Atom & Residue & Distance ( $\AA$ ) \\
\hline C2 & Ser8.A OG & 3.43 \\
\hline N3 & Ser8.A OG & 2.86 \\
\hline N3 & Ser8.A CB & 3.43 \\
\hline $\mathrm{C} 4$ & Phe116.A CE1 & 3.43 \\
\hline C5 & Phe116.A CD1 & 3.41 \\
\hline N7 & HOH506.B O & 2.87 \\
\hline N7 & Phe116.A CD1 & 3.44 \\
\hline C8 & Phe102.A CD1 & 3.21 \\
\hline C8 & Phe102.A CE1 & 3.40 \\
\hline C3' & Asp12.A OD2 & 3.27 \\
\hline C5' & Asp257.A OD2 & 3.24 \\
\hline $\mathrm{C} 5$ ' & HOH575.A O & 3.39 \\
\hline $\mathrm{O} 2{ }^{\prime}$ & Asp12.A OD1 & 2.68 \\
\hline O2' & Gly48.A N & 3.01 \\
\hline $\mathrm{O} 2{ }^{\prime}$ & Phe102.A CB & 3.37 \\
\hline $\mathrm{O} 2{ }^{\prime}$ & Gly48.A CA & 3.46 \\
\hline O3' & Asp12.A OD2 & 2.47 \\
\hline O3' & Gly48.A N & 2.93 \\
\hline O3' & Asp12.A CG & 3.24 \\
\hline O3' & Asn52.A ND2 & 3.18 \\
\hline O3' & Asp12.A OD1 & 3.27 \\
\hline O4' & Val49.A CG1 & 3.21 \\
\hline O5' & Asp257.A OD2 & 2.44 \\
\hline O5' & HOH564.A O & 2.78 \\
\hline O5' & Asp257.A CG & 3.34 \\
\hline O5' & Gln172.A NE2 & 3.17 \\
\hline CS & Arg176.A NE & 3.41 \\
\hline
\end{tabular}


Table S7. Crystal data collection and refinement statistics for MtbAdoK-4.

\begin{tabular}{|c|c|}
\hline Statistic & MtbAdoK-4 \\
\hline \multicolumn{2}{|c|}{ Data collection } \\
\hline Space Group & $\mathrm{P} 4_{1}$ \\
\hline \multicolumn{2}{|c|}{ Cell Dimensions } \\
\hline a, b, c $(\AA)$ & $49.4,49.4,263.9$ \\
\hline$\alpha, \beta, \gamma\left({ }^{\circ}\right)$ & $90,90,90$ \\
\hline Resolution $(\AA)$ & $34.96-2.0(2.07-2.0)$ \\
\hline $\mathrm{R}_{\text {merge }}$ & $0.13(0.78)$ \\
\hline $\mathrm{I} / \sigma \mathrm{I}$ & $14.55(1.97)$ \\
\hline Completeness \% & $96.0(97.62)$ \\
\hline Redundancy & 6.9 \\
\hline \multicolumn{2}{|c|}{ Refinement } \\
\hline Resolution $(\AA)$ & 2.0 \\
\hline No. of reflections & 40839 \\
\hline $\mathrm{R}_{\text {work }} / \mathrm{R}_{\text {free }}$ & $0.20 / 0.23$ \\
\hline \multicolumn{2}{|c|}{ No. of atoms } \\
\hline Protein & 4675 \\
\hline Ligand & 80 \\
\hline Water & 266 \\
\hline \multicolumn{2}{|c|}{ B factors } \\
\hline Protein & 49.0 \\
\hline Ligand/ion & 49.6 \\
\hline Water & 48.0 \\
\hline \multicolumn{2}{|c|}{ rmsd } \\
\hline Bond lengths ( $\AA$ ) & 0.004 \\
\hline Bond angles $\left({ }^{\circ}\right)$ & 1.0 \\
\hline
\end{tabular}




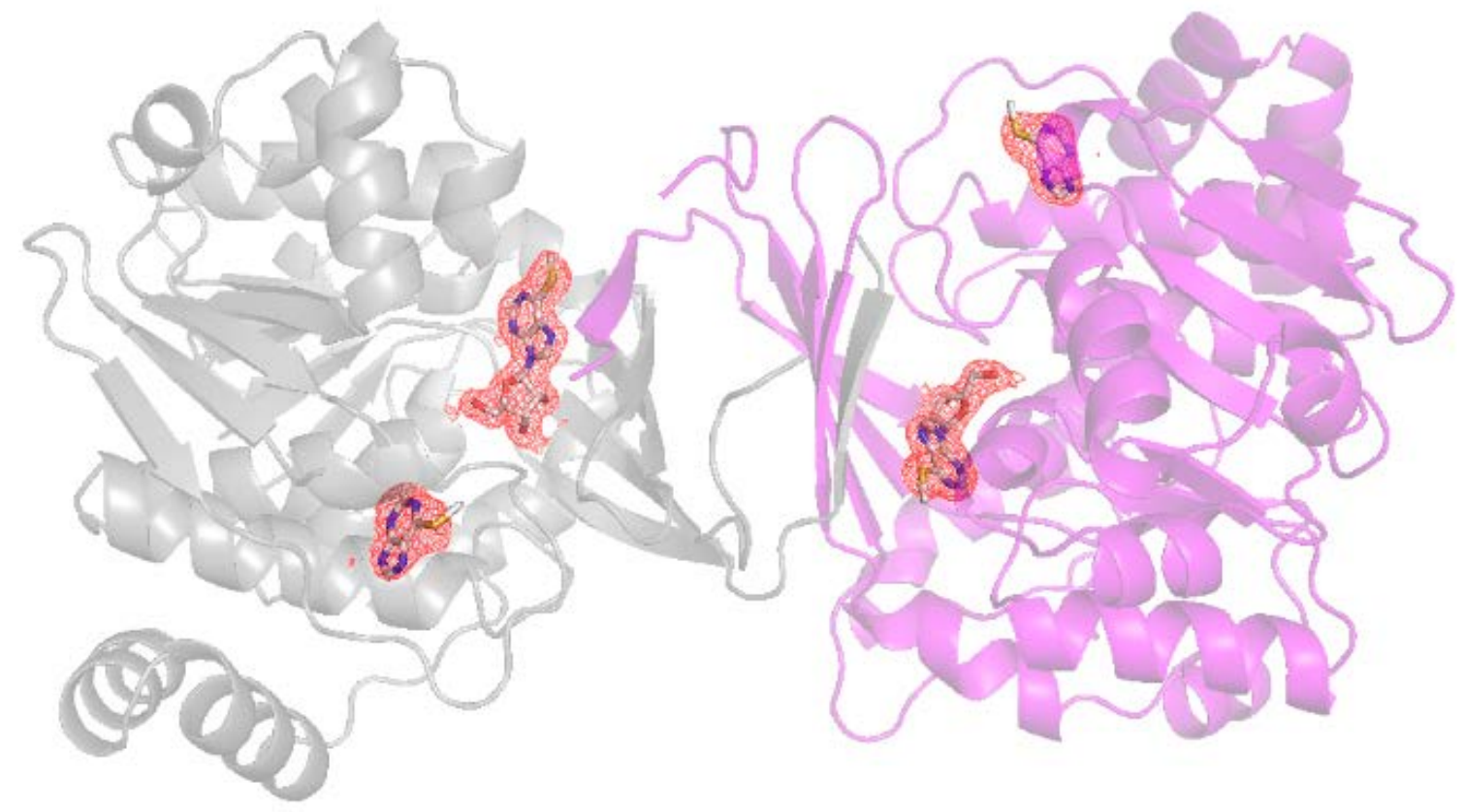

Figure S3. MtbAdoK dimer bound to two copies of compound 4 per chain. Chain A is colored gray, and chain $\mathrm{B}$ is colored magenta. $2 \mathrm{~F}_{\mathrm{o}}-\mathrm{F}_{\mathrm{C}}$ map contoured at $1.6 \sigma$.

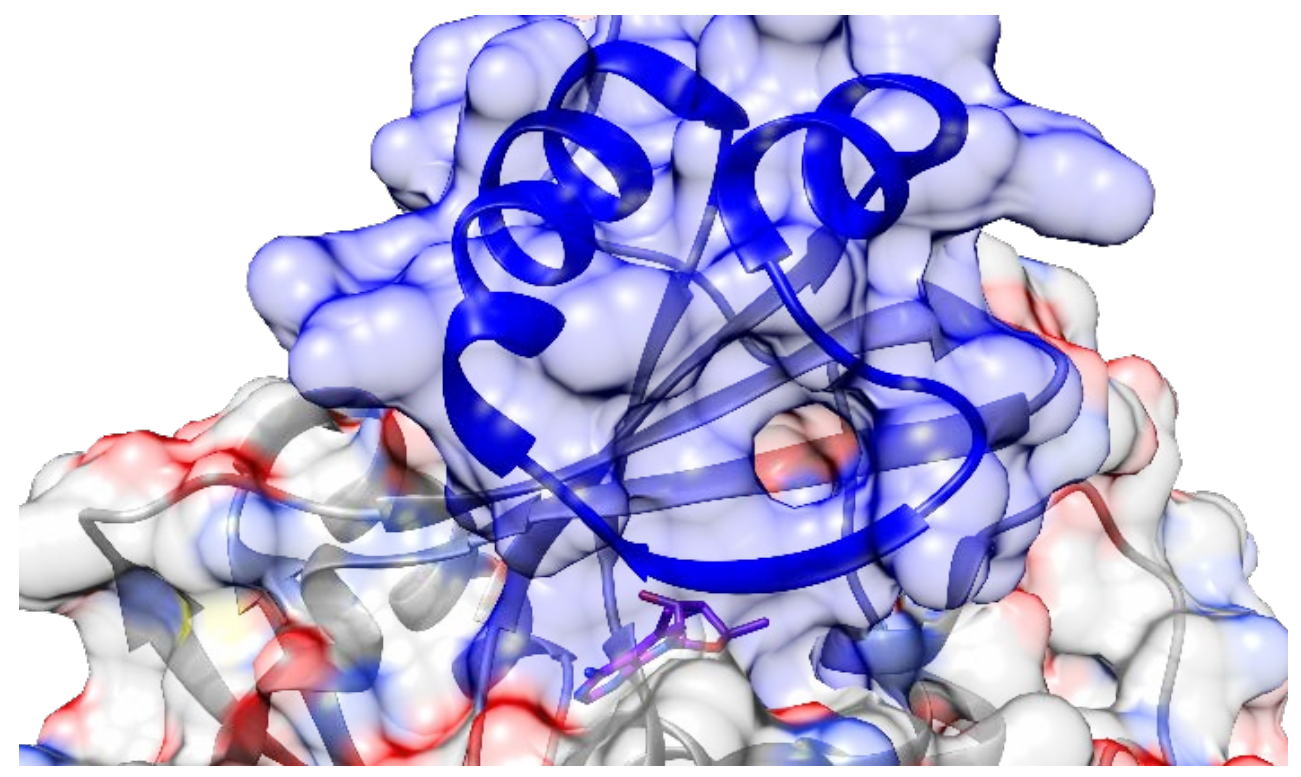

Figure S4. hAdoK-2 complex (PDB ID 2i6a). 2 (purple) is completely buried within hAdoK due to the latch-like region (blue) of the lid domain formed by residues 23-57. 


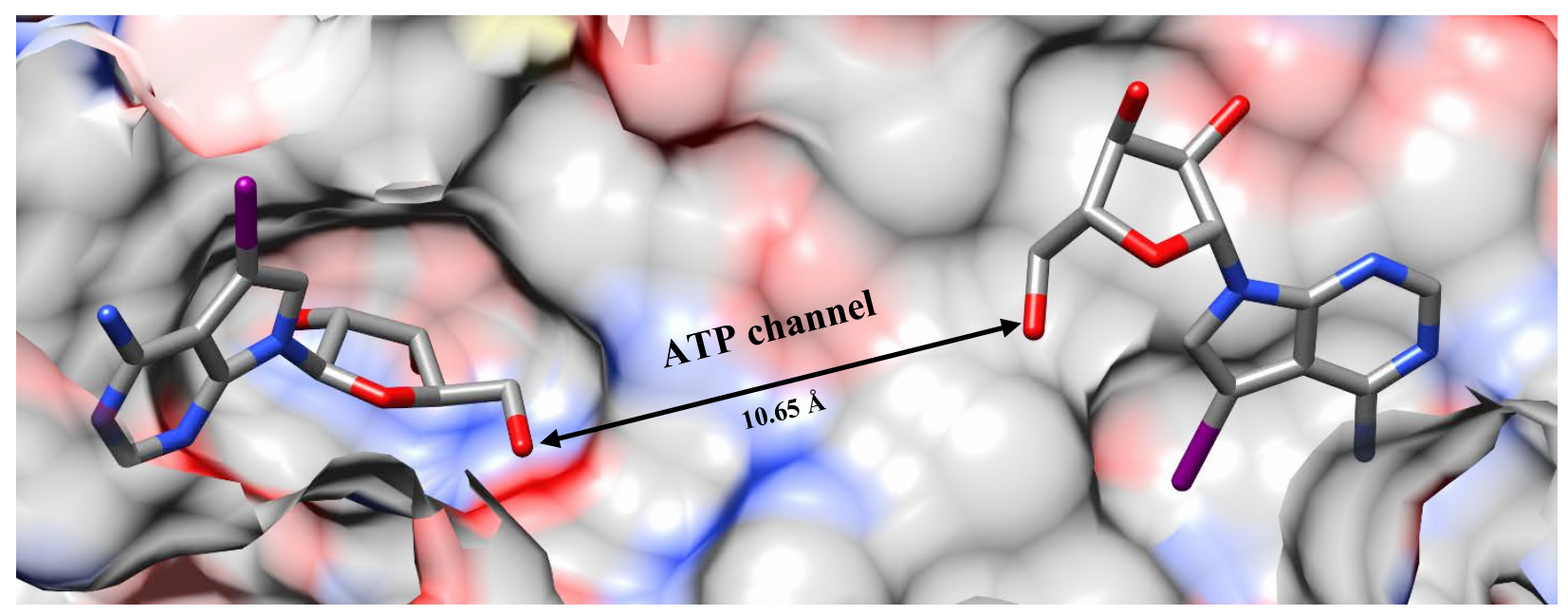

Figure S5. MtbAdoK-2 structure showing the relative position of the iodotubercidin bound to the active site (left) to that bound to the ATP site (right). The orientation and proximity of the molecules suggest that bisubstrate-like inhibitors might take advantage of both binding sites. 
Table S8. Close contacts of the MtbAdoK-5 complex $\leq 3.5 \AA$.

\begin{tabular}{|c|c|c|}
\hline Atom & Residue & Distance $(\AA)$ \\
\hline N1 & Gln173.A NE2 & 2.99 \\
\hline N1 & Gln173.A CD & 3.44 \\
\hline $\mathrm{C} 2$ & Ser8.A OG & 3.40 \\
\hline N3 & Ser8.A OG & 2.75 \\
\hline N3 & Ser8.A CB & 3.39 \\
\hline N3 & Phe116.A CE2 & 3.46 \\
\hline $\mathrm{C} 4$ & Phe116.A CD2 & 3.36 \\
\hline C4 & Phe116.A CE2 & 3.40 \\
\hline C5 & Phe116.A CD2 & 3.36 \\
\hline N6 & Gln173.A OE1 & 2.94 \\
\hline N7 & Ser36.B OG & 2.71 \\
\hline N7 & Ser36.B CB & 3.38 \\
\hline N7 & Gln172.A CG & 3.46 \\
\hline C8 & Phe102.A CE1 & 3.20 \\
\hline $\mathrm{C} 8$ & Phe102.A CD1 & 3.41 \\
\hline C2' & Asp12.A OD1 & 3.46 \\
\hline C3' & Asp12.A OD2 & 3.24 \\
\hline C5' & HOH584.A O & 3.18 \\
\hline C5' & Asp257.A OD2 & 3.18 \\
\hline O2' & Gly48.A N & 2.92 \\
\hline O2' & Asp12.A OD1 & 2.71 \\
\hline $\mathrm{O} 2{ }^{\prime}$ & Gly48.A CA & 3.33 \\
\hline O3' & Asp12.A OD2 & 2.50 \\
\hline O3' & Gly48.A N & 2.98 \\
\hline O3' & Asp12.A CG & 3.23 \\
\hline O3' & Asn52.A ND2 & 3.09 \\
\hline O3' & Asp12.A OD1 & 3.23 \\
\hline O4' & Val49.A CG1 & 3.26 \\
\hline N5' & Asp257.A OD2 & 2.61 \\
\hline N5' & HOH607.A O & 2.82 \\
\hline N5' & HOH611.A O & 2.85 \\
\hline N5' & Asp257.A CG & 3.37 \\
\hline N5' & HOH584.A O & 3.02 \\
\hline
\end{tabular}


Table S9. Crystal data collection and refinement statistics for MtbAdoK-5.

\begin{tabular}{|c|c|}
\hline Statistic & MtbAdoK-5 \\
\hline \multicolumn{2}{|c|}{ Data collection } \\
\hline Space Group & $\mathrm{P} 3_{1} 2_{1}$ \\
\hline \multicolumn{2}{|c|}{ Cell Dimensions } \\
\hline a, b, c $(\AA)$ & $71.9,71.9,110.2$ \\
\hline$\alpha, \beta, \gamma\left({ }^{\circ}\right)$ & $90,90,120$ \\
\hline Resolution $(\AA)$ & $41.2-1.9(2.02-1.95)$ \\
\hline $\mathrm{R}_{\text {merge }}$ & $0.07(0.16)$ \\
\hline $\mathrm{I} / \sigma \mathrm{I}$ & $40.2(17.16)$ \\
\hline Completeness \% & $99.8(99.92)$ \\
\hline Redundancy & 10.7 \\
\hline \multicolumn{2}{|c|}{ Refinement } \\
\hline Resolution $(\AA)$ & 1.95 \\
\hline No. of reflections & 24596 \\
\hline $\mathrm{R}_{\text {work }} / \mathrm{R}_{\text {free }}$ & $0.18 / 0.20$ \\
\hline \multicolumn{2}{|c|}{ No. of atoms } \\
\hline Protein & 2483 \\
\hline Ligand & 19 \\
\hline Water & 206 \\
\hline \multicolumn{2}{|c|}{ B factors } \\
\hline Protein & 34.1 \\
\hline Ligand/ion & 17.8 \\
\hline Water & 36.0 \\
\hline \multicolumn{2}{|c|}{ rmsd } \\
\hline Bond lengths ( $\AA$ ) & 0.009 \\
\hline Bond angles $\left({ }^{\circ}\right)$ & 1.2 \\
\hline
\end{tabular}




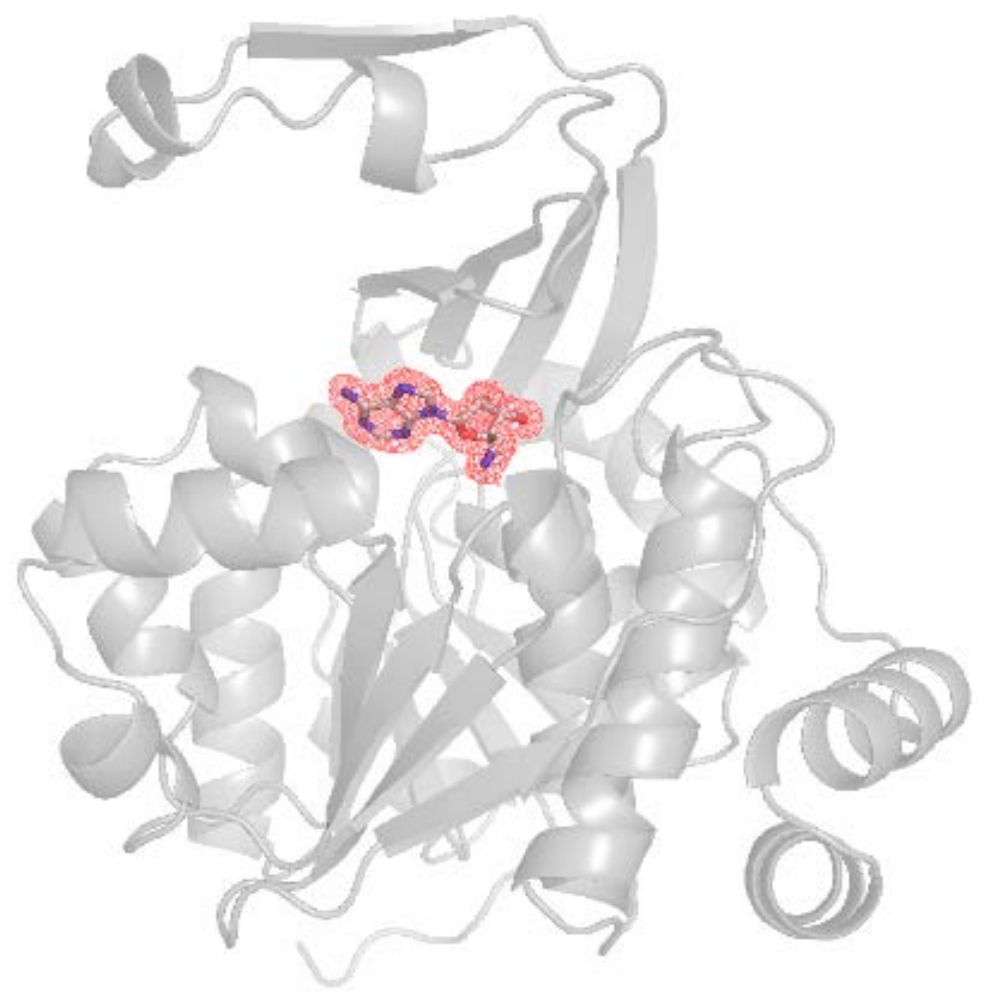

Figure S6. MtbAdoK bound to 5. Only MtbAdoK monomer observed in asymmetric unit shown. $2 \mathrm{~F}_{\mathrm{o}}-\mathrm{F}_{\mathrm{c}}$ map contoured at $1.6 \sigma$. 
Table S10. Close contacts of the MtbAdoK-6 complex $\leq 3.5 \AA$.

\begin{tabular}{|c|c|c|}
\hline Atom & Residue & Distance ( $(\AA)$ \\
\hline $\mathrm{C} 2$ & Ser8.A OG & 3.47 \\
\hline N3 & Ser8.A OG & 2.91 \\
\hline N3 & Ser8.A CB & 3.49 \\
\hline $\mathrm{C} 4$ & Phe116.A CE1 & 3.47 \\
\hline C5 & Phe116.A CD1 & 3.47 \\
\hline $\mathrm{C} 8$ & Phe102.A CD1 & 3.36 \\
\hline C8 & Phe102.A CE1 & 3.47 \\
\hline N9 & HOH563.A O & 3.49 \\
\hline $\mathrm{C} 2^{\prime}$ & Asp12.A OD1 & 3.38 \\
\hline C3' & Asp12.A OD2 & 3.23 \\
\hline $\mathrm{C} 5$, & Asp257.A OD2 & 3.27 \\
\hline C5' & HOH563.А O & 3.48 \\
\hline $\mathrm{O} 2^{\prime}$ & Asp12.A OD1 & 2.64 \\
\hline $\mathrm{O} 2^{\prime}$ & Gly48.A N & 2.96 \\
\hline $\mathrm{O} 2{ }^{\prime}$ & Gly48.A CA & 3.38 \\
\hline $\mathrm{O} 2{ }^{\prime}$ & Ala10.A CB & 3.48 \\
\hline O3' & Asp12.A OD2 & 2.51 \\
\hline O3' & Gly48.A N & 2.91 \\
\hline O3' & Asp12.A CG & 3.22 \\
\hline O3' & Asn52.A ND2 & 3.16 \\
\hline O3' & Asp12.A OD1 & 3.16 \\
\hline O4' & HOH563.A O & 2.73 \\
\hline O4' & Val49.A CG1 & 3.35 \\
\hline O5' & HOH505.A O & 2.36 \\
\hline O5' & Asp257.A OD2 & 2.64 \\
\hline O5' & Asp257.A CG & 3.37 \\
\hline O5' & HOH563.A O & 3.06 \\
\hline O5' & HOH552.А O & 3.30 \\
\hline S21 & Arg176.A CD & 3.31 \\
\hline $\mathrm{C} 22$ & Ser36.B OG & 3.22 \\
\hline C23 & Phe116.A CB & 3.45 \\
\hline C23 & Ser36.B OG & 3.38 \\
\hline
\end{tabular}


Table S11. Crystal data collection and refinement statistics for MtbAdoK-6.

\begin{tabular}{|c|c|}
\hline Statistic & MtbAdoK-6 \\
\hline \multicolumn{2}{|c|}{ Data collection } \\
\hline Space Group & $\mathrm{P} 2{ }_{1} 2_{1} 2_{1}$ \\
\hline \multicolumn{2}{|c|}{ Cell Dimensions } \\
\hline a, b, c $(\AA)$ & $75.2,81.9,158.3$ \\
\hline$\alpha, \beta, \gamma\left({ }^{\circ}\right)$ & $90,90,90$ \\
\hline Resolution $(\AA)$ & $44.38-2.10(2.17-2.10)$ \\
\hline $\mathrm{R}_{\text {merge }}$ & $0.05(0.31)$ \\
\hline $\mathrm{I} / \sigma \mathrm{I}$ & $11.89(3.43)$ \\
\hline Completeness \% & $99.7(97.90)$ \\
\hline Redundancy & 6.6 \\
\hline \multicolumn{2}{|c|}{ Refinement } \\
\hline Resolution $(\AA)$ & 2.10 \\
\hline No. of reflections & 57583 \\
\hline $\mathrm{R}_{\text {work }} / \mathrm{R}_{\text {free }}$ & $0.18 / 0.20$ \\
\hline \multicolumn{2}{|c|}{ No. of atoms } \\
\hline Protein & 5058 \\
\hline Ligand & 82 \\
\hline Water & 276 \\
\hline \multicolumn{2}{|c|}{ B factors } \\
\hline Protein & 39.6 \\
\hline Ligand/ion & 42.9 \\
\hline Water & 41.6 \\
\hline \multicolumn{2}{|c|}{ rmsd } \\
\hline Bond lengths $(\AA)$ & 0.008 \\
\hline Bond angles $\left({ }^{\circ}\right)$ & 1.14 \\
\hline
\end{tabular}




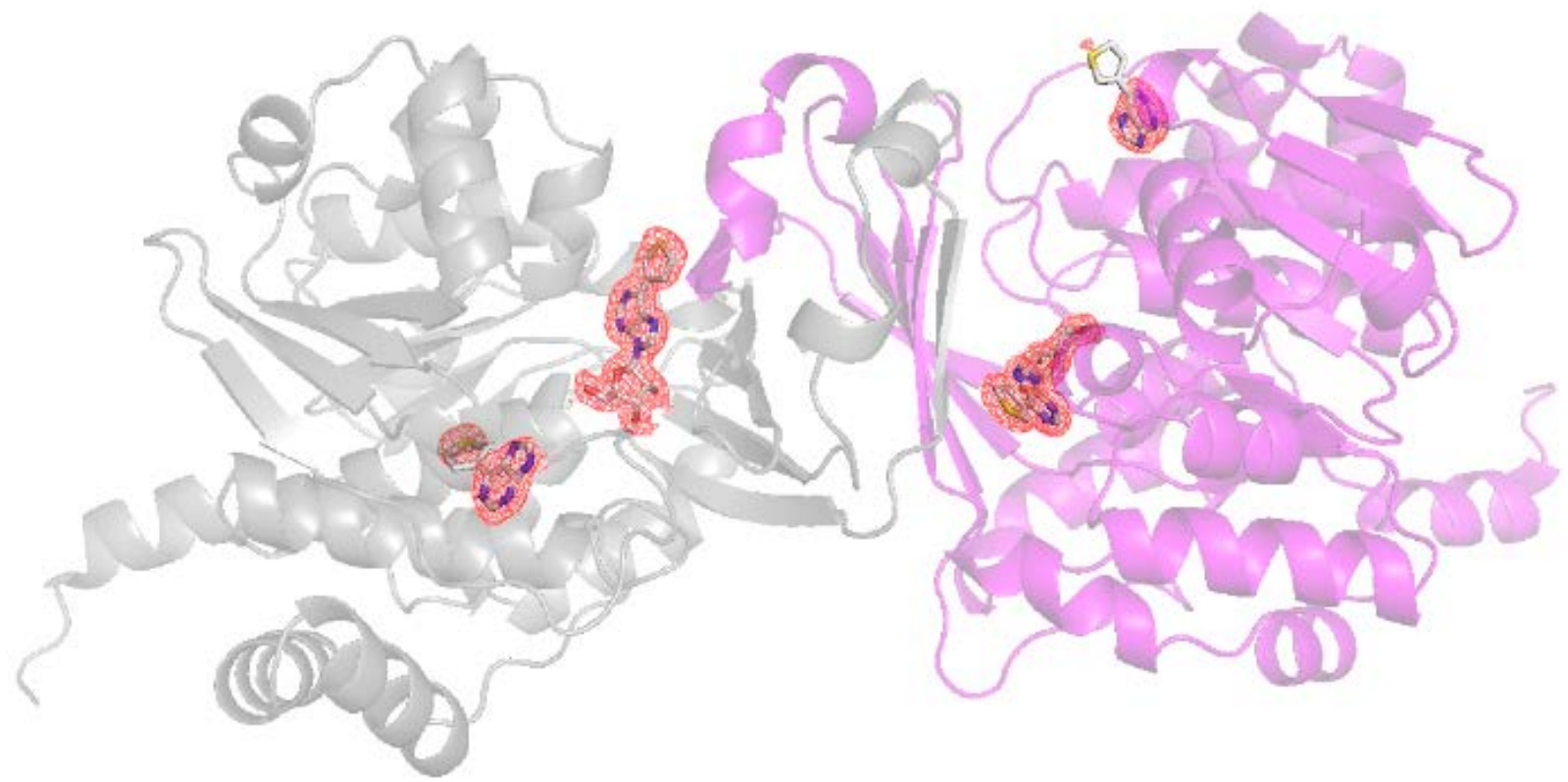

Figure S7. MtbAdoK dimer with two molecules of compound 6 per chain. Chain A is colored gray, and chain $\mathrm{B}$ is colored magenta. $2 \mathrm{~F}_{\mathrm{o}}-\mathrm{F}_{\mathrm{c}}$ maps contoured at $1.6 \sigma$. 


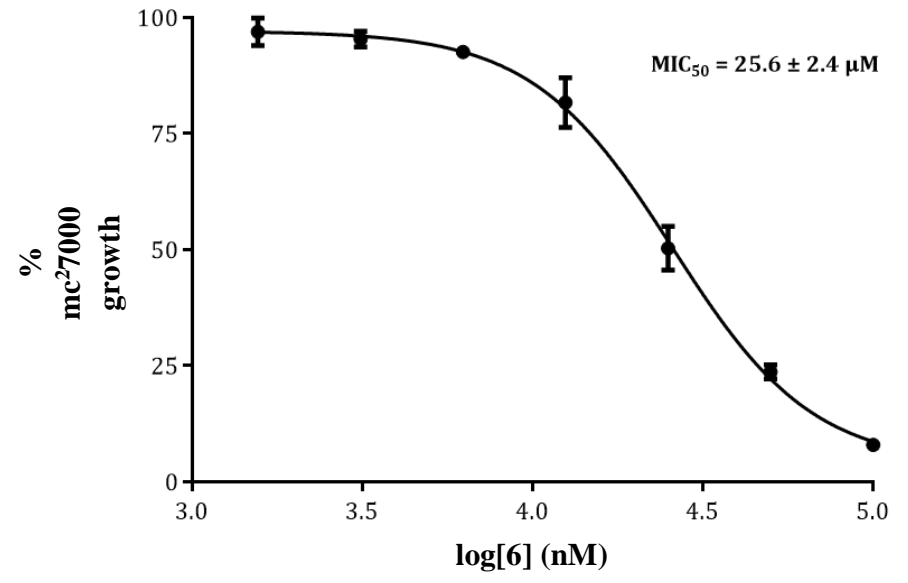

Figure S8. Dose-response curve of compound 6 when tested against $\mathrm{mc}^{2} 7000$. Data is normalized to the $0 \mu \mathrm{M}$ (DMSO) control and the error reported represents the \pm SD of 3 experiments.

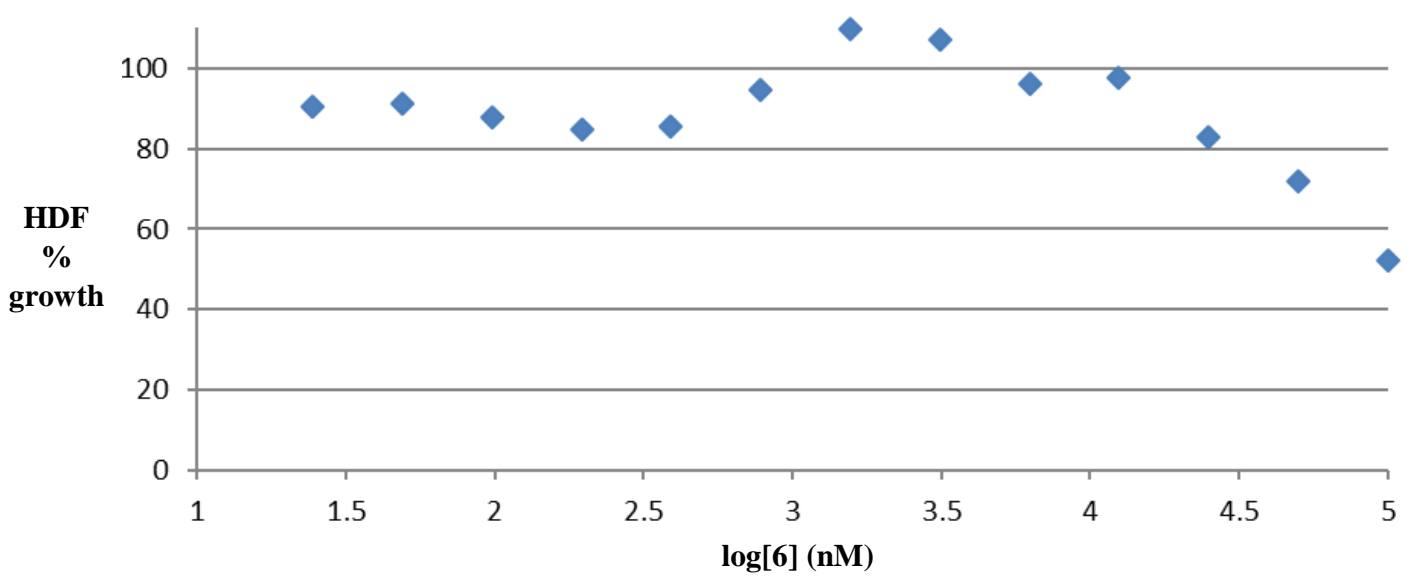

Figure S9. Dose-response curve of compound 6 when tested against HDF cells. Data is normalized to the $0 \mu \mathrm{M}$ (DMSO) control. 
Table S12. Close contacts of the MtbAdoK-7 complex $\leq 3.5 \AA$.

\begin{tabular}{|c|c|c|}
\hline Atom & Residue & Distance $(\AA)$ \\
\hline $\mathrm{C} 2$ & Ser8.A OG & 3.37 \\
\hline N3 & Ser8.A OG & 2.84 \\
\hline N3 & Ser8.A CB & 3.48 \\
\hline N7 & HOH583.B O & 2.95 \\
\hline C8 & Phe102.A CD1 & 3.32 \\
\hline C8 & Phe102.A CE1 & 3.48 \\
\hline C8 & HOH583.B & 3.48 \\
\hline $\mathrm{C} 2{ }^{\prime}$ & Asp12.A OD1 & 3.47 \\
\hline C3' & Asp12.A OD2 & 3.30 \\
\hline $\mathrm{C} 5$ ' & Asp257.A OD2 & 3.30 \\
\hline C5' & HOH694.A O & 3.49 \\
\hline $\mathrm{O} 2{ }^{\prime}$ & Asp12.A OD1 & 2.68 \\
\hline $\mathrm{O} 2{ }^{\prime}$ & Gly48.A N & 3.08 \\
\hline $\mathrm{O} 2{ }^{\prime}$ & Gly48.A CA & 3.45 \\
\hline $\mathrm{O} 2^{\prime}$ & Ala10.A CB & 3.46 \\
\hline O3' & Asp12.A OD2 & 2.48 \\
\hline O3' & Gly48.A N & 3.02 \\
\hline O3' & Asp12.A CG & 3.27 \\
\hline O3' & Asn52.A ND2 & 3.14 \\
\hline O4' & Gln172.A NE2 & 3.03 \\
\hline O4' & Val49.A CG1 & 3.51 \\
\hline O5' & Asp257.A OD2 & 2.50 \\
\hline O5' & HOH636.А O & 2.85 \\
\hline O5' & Asp257.A CG & 3.28 \\
\hline O5' & Gln172.A NE2 & 3.29 \\
\hline O5' & HOH549.A O & 3.23 \\
\hline C23 & Arg176.A NH2 & 3.48 \\
\hline C23 & Gln173.A OE1 & 3.37 \\
\hline C26 & Gln172.A O & 3.14 \\
\hline $\mathrm{C} 27$ & Gln172.A O & 3.27 \\
\hline C29 & Ser36.B O & 3.34 \\
\hline
\end{tabular}


Table S13. Crystal data collection and refinement statistics for MtbAdoK-7.

\begin{tabular}{|c|c|}
\hline Statistic & MtbAdoK-7 \\
\hline \multicolumn{2}{|c|}{ Data collection } \\
\hline Space Group & $\mathrm{P} 4_{1}$ \\
\hline \multicolumn{2}{|c|}{ Cell Dimensions } \\
\hline a, b, c $(\AA)$ & $50.0,50.0,264.6$ \\
\hline$\alpha, \beta, \gamma\left({ }^{\circ}\right)$ & $90,90,90$ \\
\hline Resolution $(\AA)$ & $43.56-1.70(1.76-1.70)$ \\
\hline $\mathrm{R}_{\text {merge }}$ & $0.09(0.51)$ \\
\hline $\mathrm{I} / \sigma \mathrm{I}$ & $11.01(2.22)$ \\
\hline Completeness \% & $99.8(98.94)$ \\
\hline Redundancy & 5.1 \\
\hline \multicolumn{2}{|c|}{ Refinement } \\
\hline Resolution $(\AA)$ & 1.70 \\
\hline No. of reflections & 70903 \\
\hline $\mathrm{R}_{\text {work }} / \mathrm{R}_{\text {free }}$ & $16.9 / 20.3$ \\
\hline \multicolumn{2}{|c|}{ No. of atoms } \\
\hline Protein & 4694 \\
\hline Ligand & 78 \\
\hline Water & 569 \\
\hline \multicolumn{2}{|c|}{ B factors } \\
\hline Protein & 25.8 \\
\hline Ligand/ion & 27.4 \\
\hline Water & 33.9 \\
\hline \multicolumn{2}{|c|}{ rmsd } \\
\hline Bond lengths $(\AA)$ & 0.010 \\
\hline Bond angles $\left({ }^{\circ}\right)$ & 1.37 \\
\hline
\end{tabular}




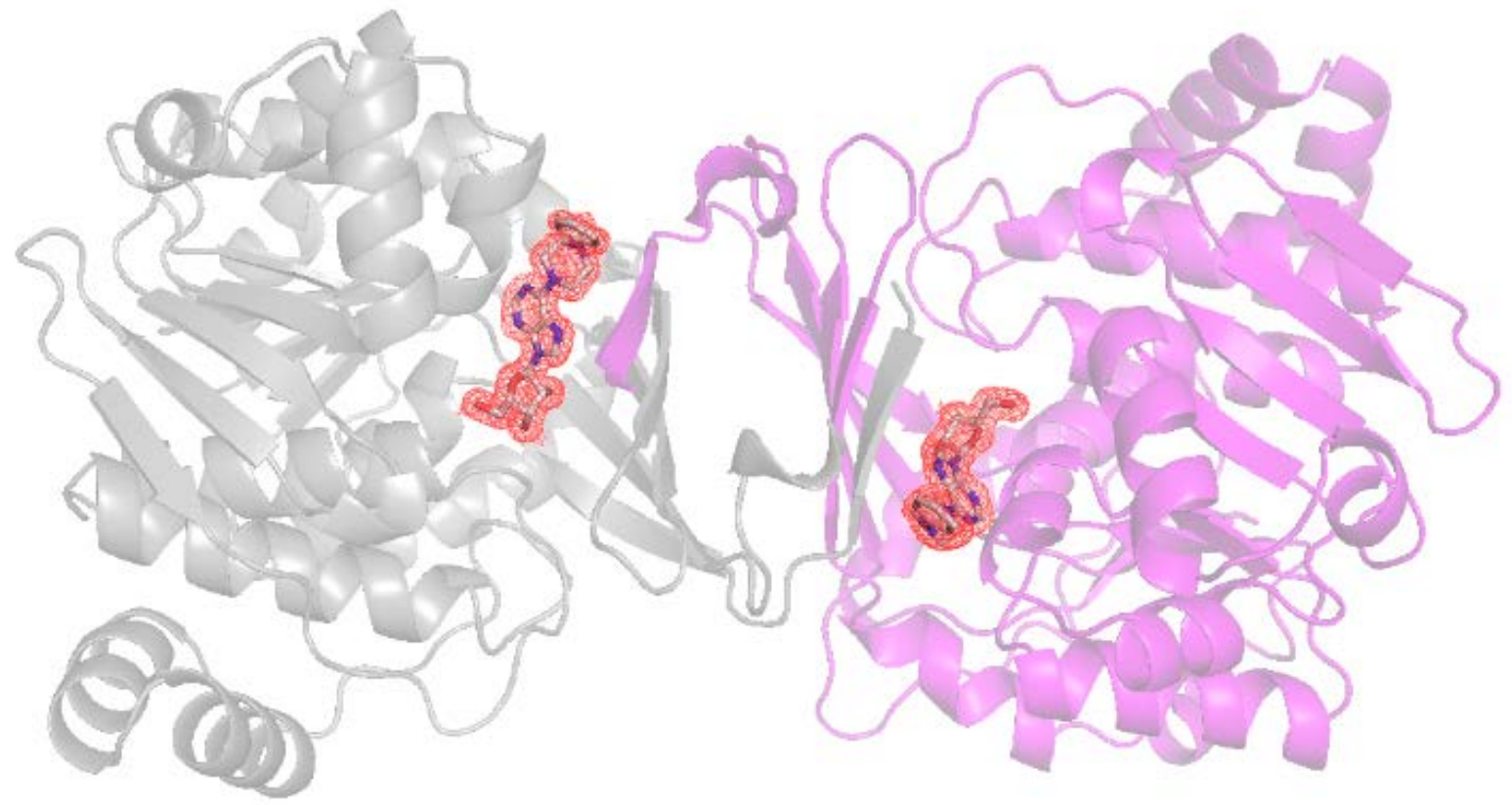

Figure S10. MtbAdoK dimer with two molecules of compound 7 per chain. Chain A is colored gray, and chain $\mathrm{B}$ is colored magenta. $2 \mathrm{~F}_{\mathrm{o}}-\mathrm{F}_{\mathrm{c}}$ maps contoured at $1.6 \sigma$. 


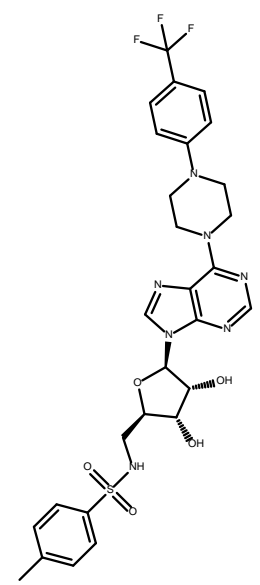

19

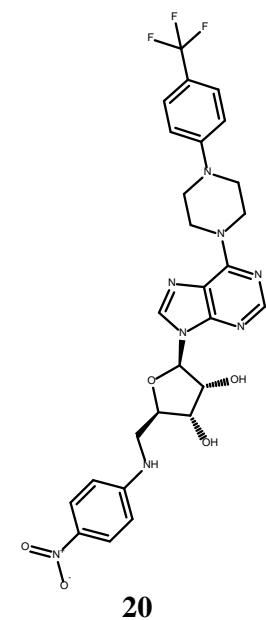

20

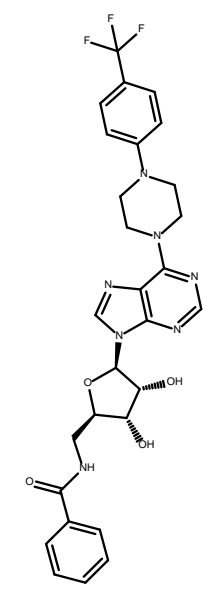

21

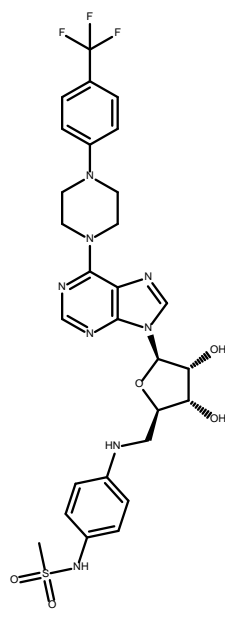

22

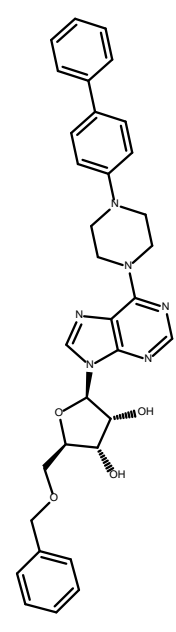

23

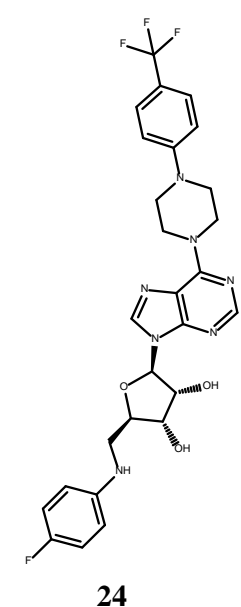

24

Figure S11. Chemical structures of synthesized adenosine analogs with substitutions at the 5'position.

Table S14. SAR data for synthesized adenosine analogs with substitutions at the 5'-position.

\begin{tabular}{|c|c|c|}
\hline ID & $\begin{array}{c}\text { MtbAdoK } \\
\text { IC } \\
(\mathbf{H M})\end{array}$ & $\begin{array}{c}\text { Mtb EC } \\
(\mathbf{\mu M})\end{array}$ \\
\hline $\mathbf{1 9}$ & $\geq 12.5$ & $>50.0$ \\
\hline $\mathbf{2 0}$ & $>50.0$ & $\geq 50.0$ \\
\hline $\mathbf{2 1}$ & $>50.0$ & $>50.0$ \\
\hline $\mathbf{2 2}$ & $>50.0$ & $>50.0$ \\
\hline $\mathbf{2 3}$ & $>50.0$ & $>50.0$ \\
\hline $\mathbf{2 4}$ & $>50.0$ & $>50.0$ \\
\hline
\end{tabular}


Table S15. Close contacts of the MtbAdoK-17 complex $\leq 3.5 \AA$.

\begin{tabular}{|c|c|c|}
\hline Atom & Residue & Distance $(\AA)$ \\
\hline N1 & Gln173.A NE2 & 3.21 \\
\hline $\mathrm{C} 2$ & Ser8.A CB & 3.45 \\
\hline $\mathrm{C} 2$ & Ser8.A OG & 3.29 \\
\hline N3 & Ser8.A OG & 2.73 \\
\hline N3 & Ser8.A CB & 3.35 \\
\hline C4 & Phe116.A CE1 & 3.39 \\
\hline $\mathrm{C} 5$ & Phe116.A CD1 & 3.46 \\
\hline N7 & Phe116.A CD1 & 3.42 \\
\hline $\mathrm{C} 8$ & Phe102.A CD1 & 3.40 \\
\hline C2' & Asp12.A OD1 & 3.30 \\
\hline C3’ & Asp12.A OD2 & 3.35 \\
\hline C5 & Asp257.A OD2 & 3.45 \\
\hline $\mathrm{O} 2^{\prime}$ & Gly48.A N & 2.88 \\
\hline $\mathrm{O} 2^{\prime}$ & Asp12.A OD1 & 2.71 \\
\hline $\mathrm{O} 2^{\prime}$ & Gly48.A CA & 3.26 \\
\hline O3’ & Asp12.A OD2 & 2.46 \\
\hline O3’ & Asp12.A CG & 3.23 \\
\hline O3’ & Gly48.A N & 3.05 \\
\hline O3’ & Asn52.A ND2 & 3.17 \\
\hline O4' & Val49.A CG1 & 3.40 \\
\hline O5' & Asp257.A OD2 & 2.36 \\
\hline O5’ & Asp257.A CG & 3.35 \\
\hline O5' & HOH589.A O & 3.14 \\
\hline $\mathrm{C} 24$ & Leu38.B CB & 3.47 \\
\hline C25 & Leu38.B N & 3.43 \\
\hline C26 & Ser36.B OG & 3.23 \\
\hline
\end{tabular}


Table S16. Crystal data collection and refinement statistics for MtbAdoK-17.

\begin{tabular}{|c|c|}
\hline Statistic & MtbAdoK-17 \\
\hline \multicolumn{2}{|c|}{ Data collection } \\
\hline Space Group & $\mathrm{P} 4_{1}$ \\
\hline \multicolumn{2}{|c|}{ Cell Dimensions } \\
\hline a, b, c $(\AA)$ & $49.9,49.9,264.3$ \\
\hline$\alpha, \beta, \gamma\left({ }^{\circ}\right)$ & $90,90,90$ \\
\hline Resolution $(\AA)$ & $35.29-2.23(2.31-2.23)$ \\
\hline $\mathrm{R}_{\text {merge }}$ & $0.06(0.16)$ \\
\hline $\mathrm{I} / \sigma \mathrm{I}$ & $26.43(10.31)$ \\
\hline Completeness \% & $98.8(98.29)$ \\
\hline Redundancy & 6.9 \\
\hline \multicolumn{2}{|c|}{ Refinement } \\
\hline Resolution $(\AA)$ & 2.23 \\
\hline No. of reflections & 30978 \\
\hline $\mathrm{R}_{\text {work }} / \mathrm{R}_{\text {free }}$ & $0.19 / 0.23$ \\
\hline \multicolumn{2}{|c|}{ No. of atoms } \\
\hline Protein & 4675 \\
\hline Ligand & 76 \\
\hline Water & 207 \\
\hline \multicolumn{2}{|c|}{ B factors } \\
\hline Protein & 47.3 \\
\hline Ligand/ion & 49.4 \\
\hline Water & 42.5 \\
\hline \multicolumn{2}{|c|}{ rmsd } \\
\hline Bond lengths $(\AA)$ & 0.004 \\
\hline Bond angles $\left({ }^{\circ}\right)$ & 0.99 \\
\hline
\end{tabular}




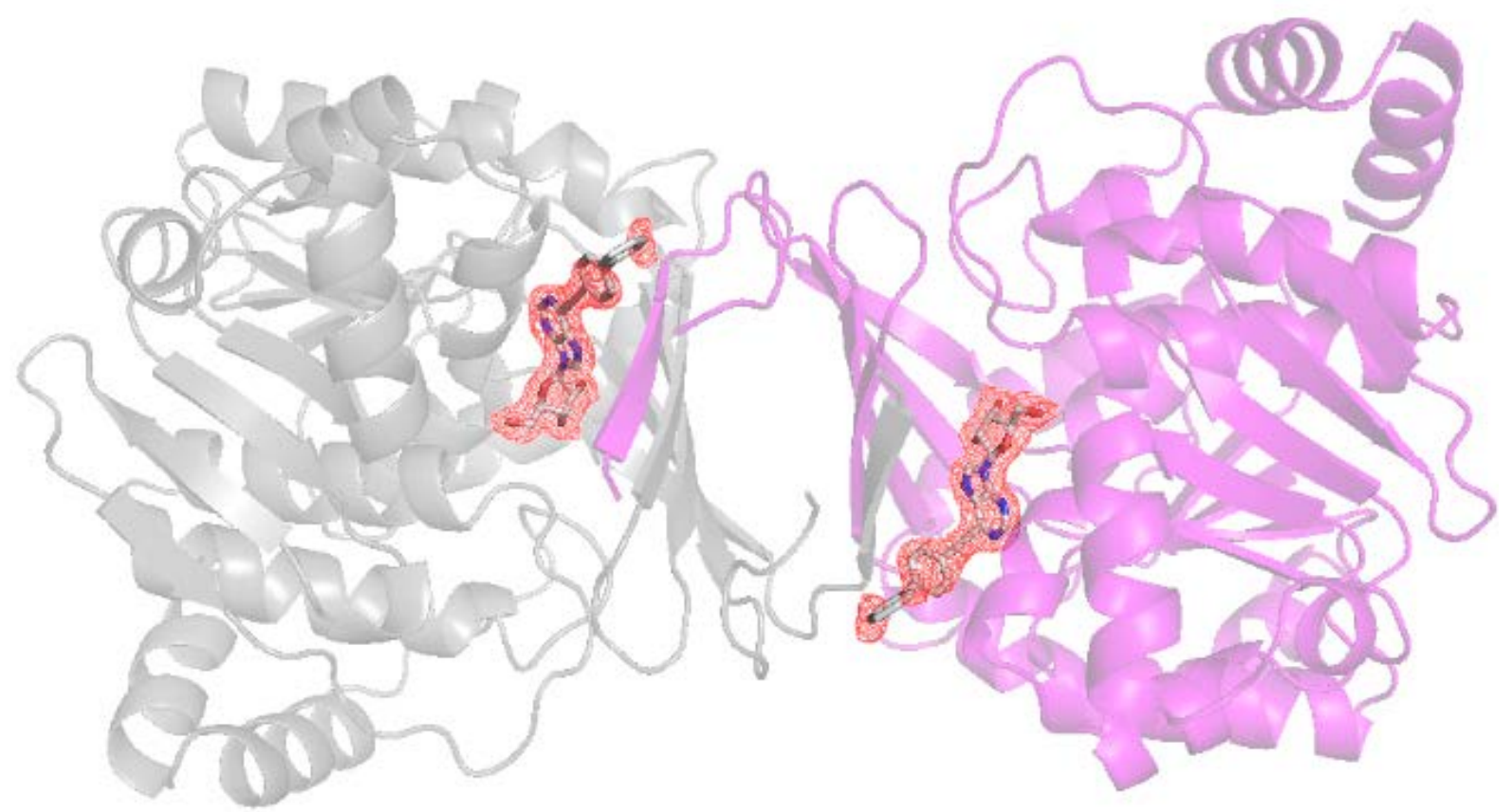

Figure S12. MtbAdoK dimer with two molecules of compound $\mathbf{1 7}$ per chain. Chain A is colored gray, and chain $\mathrm{B}$ is colored magenta. $2 \mathrm{~F}_{\mathrm{o}}-\mathrm{F}_{\mathrm{c}}$ maps contoured at $1.6 \sigma$. 
a

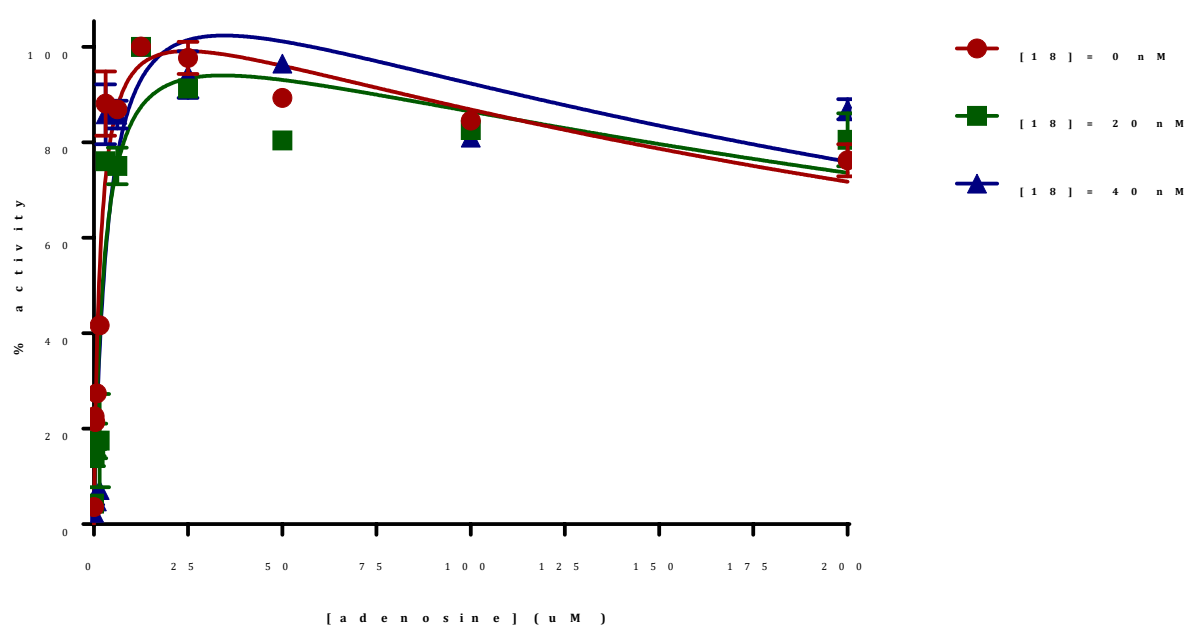

b

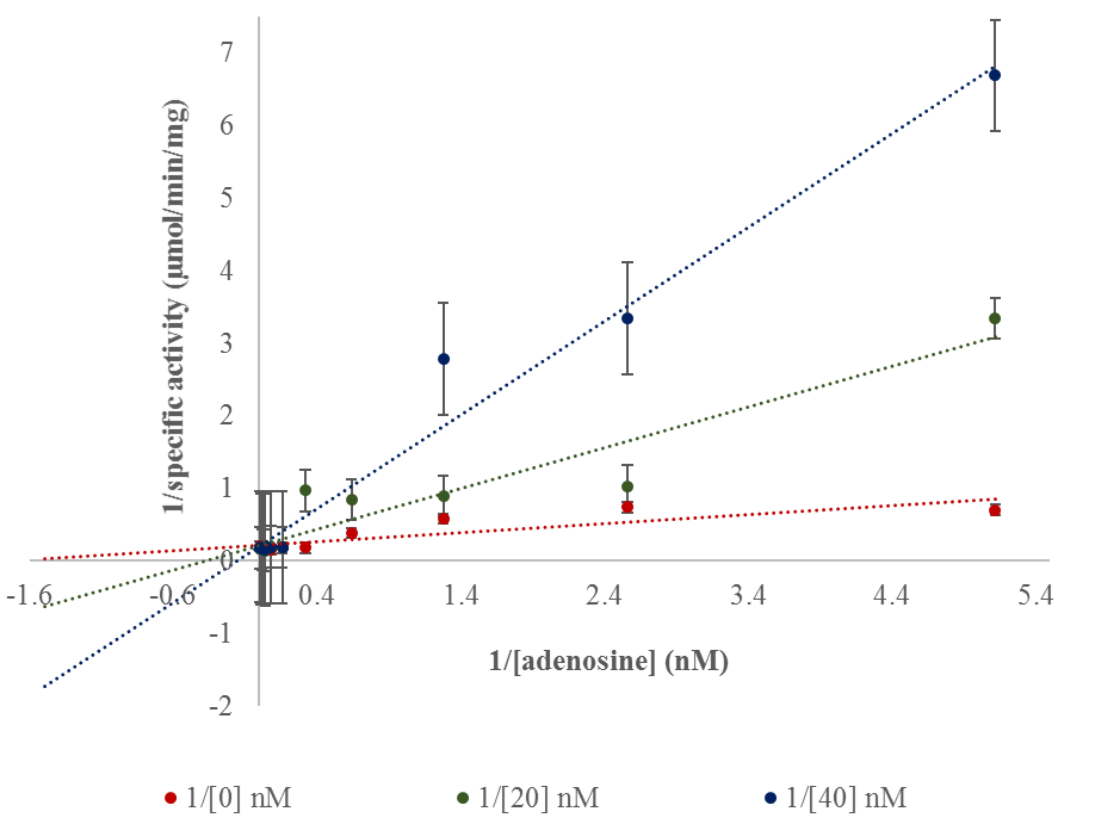

Figure S13. Steady-state kinetics for compound 18. (a) The initial velocity of MtbAdoK was plotted against increasing concentrations of adenosine in the presence of $0 \mathrm{nM}$ (DMSO-maroon), $20 \mathrm{nM}$ (green) and $40 \mathrm{nM}$ (blue) of 18. (b) Initial velocity data was transformed to linear analysis to evaluate inhibitor type. In both cases, the error bars represent \pm SD of 2 experiments.

Table S17. Steady-state kinetic parameters for MtbAdoK vs. compound 18.

\begin{tabular}{|c|c|}
\hline$[\mathbf{1 8}] \mathbf{( n M )}$ & $\mathbf{K}_{\mathbf{m}} \mathbf{( \mu \mathbf { M } )}$ \\
\hline 0.0 & $1.7 \pm 0.02$ \\
\hline 20.0 & $2.7 \pm 0.6$ \\
\hline 40.0 & $3.6 \pm 0.02$ \\
\hline
\end{tabular}

The error is reported as \pm SD of 2 experiments. 
Table S18. Mouse Acute Tolerability Studies. *

\begin{tabular}{|c|c|c|c|c|c|}
\hline \multicolumn{2}{|c|}{ Compound 18 Acute Tolerability } & \multicolumn{4}{|c}{ Mass (g) } \\
\hline Dose Level mg $\cdot \mathrm{kg}^{-1}$ & Mouse ID & Start & Day 1 & Day 2 & Day 3 \\
\hline \multirow{2}{*}{50} & Cp18-10 & 28.0 & 28.1 & 28.0 & 28.1 \\
\hline & Cp18-11 & 30.8 & 30.7 & 30.5 & 30.7 \\
\hline & Cp18-12 & 26.2 & 26.3 & 26.4 & 26.4 \\
\hline & Average & $\mathbf{2 8 . 3}$ & $\mathbf{2 8 . 4}$ & $\mathbf{2 8 . 3}$ & $\mathbf{2 8 . 4}$ \\
\hline \multirow{2}{*}{100} & Cp18-13 & 32.2 & 31.1 & 30.9 & 31.3 \\
\hline \multirow{2}{*}{200} & Cp18-14 & 28.6 & 28.7 & 28.8 & 28.8 \\
\hline & Cp18-15 & 25.0 & 25.1 & 25.0 & 25.2 \\
\hline & Average & $\mathbf{2 8 . 6}$ & $\mathbf{2 8 . 3}$ & $\mathbf{2 8 . 2}$ & $\mathbf{2 8 . 4}$ \\
\hline & Cp18-16 & 30.3 & 30.3 & 30.2 & 30.2 \\
\hline & Cp18-17 & 25.8 & 25.6 & 25.2 & 25.3 \\
\hline & Cp18-18 & 26.0 & 25.9 & 25.8 & 25.9 \\
\hline & Average & $\mathbf{2 7 . 4}$ & $\mathbf{2 7 . 3}$ & $\mathbf{2 7 . 1}$ & $\mathbf{2 7 . 1}$ \\
\hline
\end{tabular}

${ }^{*}$ Mice ( $\mathrm{n}=3$ per cohort) were administered compound $\mathbf{1 8}$ at increasing dose levels by oral gavage for three days. Mass 24 hours after dose administration was recorded. Animals retained mass and demonstrated no adverse effects such as lack of grooming, grimacing, diarrhea, or mortality. 


\section{SUPPORTING REFERENCES}

1. Zemlicka, J.; Endo, T., O6-(4-Nitrophenyl)inosine and -Guanosine as chromogenic substrates for adenosine deaminase. Nucleosides and Nucleotides 1996, 15 (1-3), 619-629.

2. Itoh, T., Sugawara, T., Mizuno, Y, A Novel Synthesis of 1-Deazaadenosine. Heterocycles 1982, 17, 305-309.

3. Katagiri, N.; Matsuhashi, Y.; Kokufuda, H.; Takebayashi, M.; Kaneko, C., A highly efficient synthesis of the antiviral agent $(+)$-cyclaradine involving the regioselective cleavage of epoxide by neighboring participation. Tetrahedron Lett 1997, 38 (11), 1961-1964. 